Center for Astrophysics

Preprint Series No. 4193

$1 / 1+1-\therefore$

\title{
THE ULTRAVIOLET SPECTRUM OF THE JOVIAN DAYGLOW
}

Weihong Liu and A. Dalgarno

Harvard-Smithsonian Center for Astrophysics 


\title{
THE ULTRAVIOLET SPECTRUM OF THE JOVIAN DAYGLOW
}

\author{
Weihong Liu and A. Dalgarno \\ Harvard-Smithsonian Center for Astrophysics, Cambridge, MA 02138 \\ To appear in The Astrophysical Journal
}

\begin{abstract}
The ultraviolet spectra of molecular hydrogen $\mathrm{H}_{2}$ and $\mathrm{HD}$ due to solar fluorescence and photoelectron excitation are calculated and compared with the Jovian equatorial dayglow spectrum measured at $3 \AA$ resolution at solar maximum. The dayglow emission is accounted for in both brightness and spectral shape by the solar fluorescence and photoelectron excitation and requires no additional energy source. The emission is characterized by an atmospheric temperature of $530 \mathrm{~K}$ and an $\mathrm{H}_{2}$ column density of $10^{20} \mathrm{~cm}^{-2}$. The dayglow spectrum contains a cascade contribution to the Lyman band emission from high-lying $E$ and $F$ states. Its relative weakness at short wavelengths is due to both self-absorption by $\mathrm{H}_{2}$ and absorption by $\mathrm{CH}_{4}$.

Strong wavelength coincidences of solar emission lines and absorption lines of $\mathrm{H}_{2}$ and $\mathrm{HD}$ produce unique line spectra which can be identified in the dayglow spectrum. The strongest fluorescence is due to absorption of the solar Lyman- $\beta$ line at $1025.72 \AA$ by the $P(1)$ line of the $(6,0)$ Lyman band of $\mathrm{H}_{2}$ at $1025.93 \AA$. The fluorescence lines due to absorption of the solar O VI line at $1031.91 \AA$ by vibrationally excited $\mathrm{H}_{2}$ via the $Q(3)$ line of the $(1,1)$ Werner band at $1031.86 \AA$ are identified. The fluorescence lines provide a sensitive measure of the atmospheric temperature. There occurs an exact coincidence of the solar O VI line at $1031.91 \AA$ and the $R(0)$ line of the $(6,0)$ Lyman band of HD at $1031.91 \AA$, but HD on Jupiter is difficult to detect due to the dominance of the $\mathrm{H}_{2}$ emission where the HD emission is particularly strong. Higher spectral resolution and higher sensitivity may make possible such a detection. The high resolution $(0.3 \AA)$ spectra of $\mathrm{H}_{2}$ and HD are presented to stimulate search for the HD on Jupiter with the Hubble Space Telescope.
\end{abstract}

Subject headings: planets and satellites: individual (Jupiter) - ultraviolet: solar system - ultraviolet: spectra 


\section{Introduction}

Ultraviolet emission of molecular hydrogen has been detected from the atmosphere of Jupiter and other outer planets since the Voyager flybys of Jupiter in 1979. The excitation mechanism for the dayglow is still the subject of controversy. The excited electronic states of molecular hydrogen may be populated by absorption of solar radiation and photoelectron impact (Yelle 1988a) or by impact of electrons (Shemansky \& Ajello 1983; Shemansky 1985) possibly accelerated by dynamo action (Clarke, Hudson, \& Yung 1987; Hudson, Clarke, \& Warren 1989). The correlation of dayglow emissions with the solar flux supports the solar fluorescence mechanism (Yelle et al. 1987) but the dayglow emission intensities indicate that there is an additional source (Strobel et al. 1991). The controversy may be settled by determining whether the dayglow emission can be accounted for by the solar fluorescence and photoelectron excitation together or there is a significant residual intensity that must be generated by other excitation processes. Solar fluorescence and photoelectron excitation and the accelerated electron mechanisms yield similar ultraviolet spectra at the low spectral resolution of $30 \AA$ with which the earliest Jovian dayglow spectrum was obtained with the Voyager UVS. The IUE spectra of the Jovian dayglow obtained at $10 \AA$ resolution with low signal-to-noise ratios do not permit a definite conclusion about the relative importance of the mechanisms (McGrath et al. 1989). The issue may be settled by constructing theoretical models to analyze the spectrum obtained at $3 \AA$ resolution and higher sensitivity with the Hopkins Ultraviolet Telescope (HUT) (Feldman et al. 1993). A reliable solar fiuorescence model is made possible by the recent advance in the knowledge of the solar extreme ultraviolet (EUV) spectrum.

In this paper we present a theoretical model of excitation and emission of molecular hydrogen in the Jovian upper atmosphere due to absorption of solar radiation. We employ a reliable solar EUV spectrum based on recent measurements and models. We calculate the rates of population of the rovibrational levels of various excited electronic states of molecular hydrogen by solar fluorescence and photoelectron excitation. We take into account effects of the secondary electrons produced as the photoelectrons slow down. We construct synthetic ultraviolet spectra of $\mathrm{H}_{2}$ and $\mathrm{HD}$ and compare them with the Jovian equatorial dayglow spectrum. We determine the atmospheric temperature and the $\mathrm{H}_{2}$ column density at the peak of the dayglow emission. We discuss the possible detection of $\mathrm{HD}$ on Jupiter. We conclude that the Jovian dayglow emission is accounted for by a combination of solar fluorescence and photoelectron excitation, and we rule out the hypothesis of the dynamo acceleration and energetic electron excitation as an excitation mechanism for the Jovian dayglow emission. Thus we may have put an end to the decade-long controversy on the excitation mechanism for Jovian dayglow emission.

\section{The Model}

\subsection{Solar Extreme Ultraviolet Spectrum}

No direct measurements of solar EUV flux were available in December 1990 near the maximum of solar cycle 22 when the Jovian dayglow spectrum was obtained with the HUT. We estimated the solar maximum EUV flux by extrapolating the solar flux obtained in November 1988 during moderate solar activity with the San Marco satellite and rockets (Schmidtke et al. 1992) by using the solar EUV irradiance model EUV91 (Tobiska 1991, 1993). The EUV91 model was based on the solar EUV data sets obtained with satellites and rockets (Tobiska 1993 and references therein). The model was constructed using a multiple linear regression technique with four ground-based solar indices: Lyman- $\alpha$ flux and He I-1.083 $\mu$ m equivalent width scaled to Lyman- $\alpha$ for chromospheric emission, and daily and 81-day running mean values of the $10.7 \mathrm{~cm}$ flux for coronal emission. The solar flux at a wavelength $\lambda$ and at time $t$ is written

$$
F(\lambda, t)=a_{0}(\lambda)+\sum_{i=1}^{4} a_{i}(\lambda) I_{i}(t)
$$

where $a_{i}(\lambda)$ are the solar index coefficients and $I_{i}(t)$ are the solar index data sets given by Tobiska (1991).

The solar EUV spectrum at $5.27 \mathrm{AU}$ appropriate for Jupiter at the time of the HUT observations during high solar activity $\left(F_{10.7 \mathrm{~cm}}=210 \mathrm{~W} \mathrm{~m}^{-2}\right)$ is shown in Figure 1, compared with the spectra for moderate $\left(F_{10.7 \mathrm{~cm}}=150 \mathrm{~W} \mathrm{~m}^{-2}\right)$ (Schmidtke et al. 1992) and low $\left(F_{10.7 \mathrm{~cm}}=70 \mathrm{~W} \mathrm{~m}^{-2}\right)$ (Hinteregger, Pukui, \& Gilson 1981) solar activities. Solar cycle 22 is more active than the previous solar cycles (Hoegy et al. 1993). The temporal variation of the solar flux within the solar cycle is larger at shorter wavelengths than at longer wavelengths. The total integrated solar flux in the $18-500 \AA$ wavelength range which contributes to photoionization followed by photoelectron impact excitation of $\mathrm{H}_{2}$ is $1.8 \mathrm{kR}$ for high solar activity, which is a factor of 1.5 higher than the $1.3 \mathrm{kR}$ for moderate activity and a factor of 3.6 higher than the $0.51 \mathrm{kR}$ for low activity. As an important source of the photoelectrons, the solar He II line at $303.78 \AA$ has a flux of $0.28 \mathrm{kR}$ for high solar activity compared with $0.22 \mathrm{kR}$ for low activity. The He II line is the single most important line source of photoelectrons rather than the dominant source. The total integrated solar flux between $800 \AA$ and $1200 \AA$ which contributes to the solar fluorescence of $\mathrm{H}_{2}$ and $\mathrm{HD}$ is $3.6 \mathrm{kR}$ for high solar activity, a factor of 1.5 higher than the $2.4 \mathrm{kR}$ for moderate activity and a factor of 2.7 higher than the $1.3 \mathrm{kR}$ for low activity. The relative contribution to the dayglow emission by photoelectron excitation compared with solar fluorescence is significantly higher at solar maximum than at solar minimum. 
The flux of the solar Lyman- $\beta$ line at $1025.72 \AA$ is estimated to be about $8.1 \mathrm{kR}$ at $1 \mathrm{AU}$ at the solar maximum, higher than the $6 \mathrm{kR}$ assumed by Strobel et al. (1991) and Feldman et al. (1993). At Jupiter, the solar Lyman- $\beta$ flux is $0.29 \mathrm{kR}$ for high solar activity compared with $0.19 \mathrm{kR}$ for moderate activity and $0.16 \mathrm{kR}$ at solar minimum. There is a factor of 1.9 variation for the solar Lyman- $\beta$ flux during solar cycle 22 , essentially the same as the factor of 2 for solar cycle 21 (Lean 1987). The solar Lyman- $\beta$ profile was measured (Tousey et al. 1964; Lemaire et al. 1978) and it has a central absorption feature with two peaks separated by about $0.3 \AA$ with an effective width of about $0.1 \AA$ for each. The solar Lyman$\alpha$ line has a similar line profile to the solar Lyman- $\beta$ line (Lemaire et al. 1978). The profiles of other solar emission lines are uncertain. We modeled them as Gaussians with a Doppler width of $0.1 \AA$.

Yelle (1988a) based his calculations of the solar fluorescence spectrum of $\mathrm{H}_{2}$ on the Atmospheric Explorer solar minimum reference spectrum of Hinteregger et al. (1981) for solar cycle 21 and obtained a solar maximum spectrum by multiplying the solar minimum flux by a uniform factor of 3.9 for all wavelengths. The factor of 3.9 is higher than our estimate of 3.6 solar-cycle variation in the spectral region below $500 \AA$. The solar Lyman- $\beta$ flux assumed by Yelle (1988a) is $17 \mathrm{kR}$ at $1 \mathrm{AU}$, a factor of 2 higher than our estimate and the estimate by Lean (1987). The high solar Lyman- $\beta$ flux in Yelle's model leads to an overestimate of the contribution of solar fluorescence to the dayglow excitation.

\subsection{Absorption of Solar Radiation}

We consider absorption of the solar EUV flux in the Jovian upper atmosphere by a mixed gas of $\mathrm{H}_{1} \mathrm{H}_{2}$, and HD with column densities of $N(\mathrm{H}), N\left(\mathrm{H}_{2}\right)$, and $N(\mathrm{HD})$ respectively. The solar spectrum was divided into bins with size of $10^{-3} A$ and absorption of the solar radiation by overlapping lines of $\mathrm{H}, \mathrm{H}_{2}$, and $\mathrm{HD}$ was taken into account. The absorbed photon flux at wavelength $\lambda$ is given by

$$
I_{\lambda}=I_{\lambda}^{s}\left(1-e^{-\tau}\right),
$$

where $I_{\lambda}^{z}$ is the solar maximum flux at $5.27 \mathrm{AU}$ at wavelength $\lambda$, and $\tau$ is the total optical depth

$$
\tau=\sum_{c} \tau_{\lambda}^{c}+\sum_{\mathrm{S}} \sum_{l} \tau_{\lambda}^{l}(\mathrm{~S}), \quad \mathrm{S}=\mathrm{H}, \mathrm{H}_{2}, \mathrm{HD}
$$

The optical depth for continuum absorption by $\mathrm{H}_{2}$ is given by

$$
\tau_{\lambda}^{c}=\sigma_{\lambda}^{c} N\left(\mathrm{H}_{2}\right),
$$

where $\sigma_{\lambda}^{c}$ is the photoionization cross section of $\mathrm{H}_{2}$ for parent or dissociative ionization. Photoionization of $\mathrm{H}$ or HD is unimportant because of their low abundances and was neglected in the calculations. The optical depth for line absorption by $\mathrm{H}, \mathrm{H}_{2}$, or $\mathrm{HD}$ is given by

$$
\begin{aligned}
\tau_{\lambda}^{l}(\mathrm{~S}) & =N(\mathrm{~S}) \sigma_{\lambda}^{l}(\mathrm{~S}, n), & \mathrm{S}=\mathrm{H}, \\
& =N(\mathrm{~S}, v J) \sigma_{\lambda}^{l}\left(\mathrm{~S}, v J \rightarrow i v^{\prime} J^{\prime}\right), & \mathrm{S}=\mathrm{H}_{2}, \mathrm{HD},
\end{aligned}
$$

for the transition from the ground state of $H$ to an excited state $n$, or for the transition from a lower rovibrational level $v J$ of the ground electronic state of $\mathrm{B}_{2}$ or $\mathrm{HD}$ to an upper rovibrational level $v^{\prime} J^{\prime}$ of an excited electronic state $i$. In thermal equilibrium, the population of the rovibrational level $v J$ of the ground electronic state of $\mathrm{H}_{2}$ or $\mathrm{HD}$ is given by

$N(\mathrm{~S}, v J)=N(\mathrm{~S}) \frac{(2 J+1) g_{s} \exp \left(-E_{v J} / T\right)}{\sum_{v J}(2 J+1) g_{s} \exp \left(-E_{v J} / T\right)}, \mathrm{S}=\mathrm{H}_{2}, \mathrm{HD}$,

where $E_{v J}$ is the energy of the level $v J, T$ is the temperature, and $g_{s}$ is the nuclear spin statistical weight of $\mathrm{H}_{2}$ or HD which is 3 for odd rotational quantum numbers of $\mathrm{H}_{2}$ and 1 otherwise. The line absorption cross section is given by

$$
\begin{array}{rlrl}
\sigma_{\lambda}^{l} & =\frac{3 \lambda^{\star}}{8 \pi c} A(\mathrm{~S}, n) \phi_{\lambda}, & \mathrm{S}=\mathrm{H}, \\
& =\frac{\lambda^{\dagger}}{8 \pi c} A\left(\mathrm{~S}, i, v^{\prime} J^{\prime} \rightarrow v J\right) \frac{g_{J^{\prime}}}{g g} \phi_{\lambda}, & & \mathrm{S}=\mathrm{H}_{2}, \mathrm{HD},
\end{array}
$$

where $A$ is the transition probability and $g$ is the statistical weight. Absorptions from the vibrational levels $v=0,1$ of $\mathrm{H}_{2}$ and the level $v=0$ of $\mathrm{BD}$ were included. The line profile can be written

$$
\phi_{\lambda}=\frac{1}{\alpha_{D}} \sqrt{\frac{\ln 2}{\pi}} K(x, y),
$$

where the Voigt function $K(x, y)$ is given by

$$
\begin{gathered}
K(x, y)=\frac{y}{\pi} \int_{-\infty}^{\infty} \frac{\exp \left(-t^{2}\right)}{y^{2}+(x-t)^{2}} d t, \\
x=\frac{\lambda-\lambda_{0}}{\alpha_{D}} \sqrt{\ln 2}, \\
y=\frac{\alpha_{L}}{\alpha_{D}} \sqrt{\ln 2},
\end{gathered}
$$

the Lorentz width $\alpha_{L}$ by

$$
\alpha_{L}=\frac{\lambda_{0}^{2} A_{\text {tot }}}{4 \pi c},
$$

and the Doppler width $\alpha_{D}$ by

$$
\alpha_{D}=\lambda_{0} \sqrt{\frac{2 k T}{m c^{2}} \ln 2},
$$

$\lambda_{0}$ is the transition wavelength and $A_{\text {tot }}$ is the inverse of the lifetime of the upper level. The column production rate of photoelectrons is

$$
P_{p e}=I_{\lambda} \frac{\sum_{\varepsilon} \tau_{\lambda}^{c}}{\tau}
$$


The radiative excitation rate of the upper level $i v^{\prime} J^{\prime}$ of $\mathrm{H}_{2}$ or $\mathrm{HD}$ is

$$
P\left(\mathrm{~S}, v J \rightarrow i v^{\prime} J^{\prime}\right)=I_{\lambda} \frac{\tau_{\lambda}^{l}(\mathrm{~S})}{\tau}, \mathrm{S}=\mathrm{H}_{2}, \mathrm{HD} .
$$

The line transition probabilities of $\mathrm{H}$ were obtained from the calculations of Karzas \& Latter (1961). The line transition probabilities for the Lyman $B^{1} \Sigma_{u}^{+} \rightarrow X^{1} \Sigma_{g}^{+}$ and Werner $C^{1} \Pi_{u} \rightarrow X^{1} \Sigma_{g}^{+}$transitions of $\mathrm{H}_{2}$ were obtained from the calculations of Abgrall et al. (1993a,b). They differ from those obtained by utilizing the band transition probabilities calculated by Allison \& Dalgarno (1970) with the Honl-London factors when the transition dipole moment has significant dependence on the rotational quantum number or when rotational-vibrational coupling is important. Only band transition probabilities are available for other electronic transitions of $\mathrm{H}_{2}$ and for $\mathrm{HD}$, and rotational line transition probabilities were obtained from band transition probabilities by using the Honl-London factors. The band transition probabilities of $\mathrm{H}_{2}$ were obtained from Kwok, Dalgarno, \& Posen (1985) for the $B^{\prime 1} \Sigma_{u}^{+} \rightarrow X^{1} \Sigma_{g}^{+}$transition and from Glass-Maujean (1984) for the $D^{1} \Pi_{u} \rightarrow X^{1} \Sigma_{g}^{+}$transition. Given that the $B^{\prime \prime 1} \Sigma_{u}^{+}$and $D^{\prime 1} \Pi_{u}$ states have approximately the same equilibrium separation as the $B^{\prime 1} \Sigma_{u}^{+}$ and $D^{1} \Pi_{u}$ states respectively, the branching ratios for the $B^{\prime \prime 1} \Sigma_{u}^{+} \rightarrow X^{1} \Sigma_{g}^{+}$and $D^{\prime 1} \Pi_{u} \rightarrow X^{1} \Sigma_{g}^{+}$transitions were assumed to be the same as those for the $B^{\prime 1} \Sigma_{u}^{+} \rightarrow X^{1} \Sigma_{g}^{+}$ and $D^{1} \Pi_{u} \rightarrow X^{1} \Sigma_{g}^{+}$transitions respectively. The band transition probabilities for the Lyman $B^{1} \Sigma_{u}^{+} \rightarrow X^{1} \Sigma_{g}^{+}$ and Werner $C^{1} \Pi_{u} \rightarrow X^{1} \Sigma_{g}^{+}$transitions of $\mathrm{HD}$ are given by Allison \& Dalgarno (1970). The photoionization cross sections of $\mathrm{H}_{2}$ were taken from the measurements of Backx, Wight, \& Van der Wiel (1976) and Chung et al. (1993). They were also calculated by Cacelli, Moccia, \& Rizzo (1993).

The spectrum of photoelectrons resulting from photoionization of $\mathrm{H}_{2}$ in the Jovian upper atmosphere by the solar EUV photons is shown in Figure 2. The photoelectrons have energies mostly lower than $60 \mathrm{eV}$, and the average energy is $33 \mathrm{eV}$ for those energetic enough to contribute to the Lyman band emission of $\mathrm{H}_{2}$. The photoelectron flux peaks at $25 \mathrm{eV}$ due to the photoionization mostly by the strong solar He II line at $303.78 \AA$ with an energy flux of $8.4 \times 10^{9} \mathrm{eV} \mathrm{cm}^{-2} \mathrm{~s}^{-1}$, equaling about $15 \%$ of the total integrated photoelectron energy flux of $6.3 \times 10^{10} \mathrm{eV} \mathrm{cm}^{-2} \mathrm{~s}^{-1}$.

\subsection{Photoelectron Impact Excitation}

The photoelectrons lose their energy in the Jovian upper atmosphere mainly by exciting, dissociating, and ionizing $\mathrm{H}_{2}$, because of the generally low fractional ionization. The processes of electron energy deposition in $\mathrm{H}_{2}$ gas have been described in detail by Cravens, Victor, \& Dalgarno (1975), including the effects of slowing down of the secondary electrons. It had been applied to determining the cosmic-ray-induced photodestruction rates of interstellar molecules (Gredel, Lepp, \& Dalgarno 1987; Gredel et al. 1989). We present here a brief description of our computational method for electron energy deposition in an $\mathrm{H}_{2}$ gas. For the highest energy $E_{p}$ of photoelectrons a discrete set of energies $E_{1}, E_{2}, \ldots, E_{p}$ is selected such that the energy bin sizes $\Delta E_{i}=E_{i}-E_{i-1}$ are smaller than the threshold energies of all the inelastic processes that contribute significantly to the energy loss at $E_{i}$. We chose a bin size of $0.1 \mathrm{eV}$ which is smaller than the vibrational excitation threshold of $\mathrm{H}_{2}$ of $0.52 \mathrm{eV}$. The probabilities that the bin at the highest energy is emptied by excitation and ionization are computed from the electron impact cross sections. Each successive lower energy bin is emptied in turn until no inelastic process is possible and all of the remaining energy inputs as heat. The rate is recorded at which each excited or ionized state of $\mathrm{H}_{2}$ is populated. The total population of each excited or ionized state is the sum of the excitation or ionization yields in each step. The accuracy of the method is limited by the bin sizes chosen. The uncertainty is less than $1 \%$ for the bin size of $0.1 \mathrm{eV}$, much smaller than the uncertainties of the input cross sections of $\mathrm{H}_{2}$ which are at least $10 \%$.

Electron impact excitations of $\mathrm{H}_{2}$ were included to the first and second excited vibrational levels of the ground $X^{1} \Sigma_{g}^{+}$state, and to the vibrational levels of the $B^{1} \Sigma_{u}^{+}$, $B^{\prime 1} \Sigma_{u}^{+}, B^{\prime \prime} \Sigma_{u}^{+}, C^{1} \Pi_{u}, D^{1} \Pi_{u}, D^{\prime 1} \Pi_{u}$ Rydberg states, the $E, F^{1} \Sigma_{g}^{+}$and $a^{3} \Sigma_{g}^{+}$valence states, and the repulsive $b^{3} \Sigma_{u}^{+}$ state. The excitation rates of the rotational levels were obtained by assuming that the branching ratios for electron impact excitation are the same as those for radiative excitation. Pure rotational excitations are negligible energy loss channels and were not included in the calculations because of the low rotational excitation energies and because the rotational levels are in thermal equilibrium in the Jovian upper atmosphere. Excitation from the vibrationally excited levels of the ground electronic state is unimportant due to their relatively low populations.

The cross sections for electron impact vibrational excitation were obtained from the measurements of Nishimura \& Danjo (1986), and Brunger et al. (1991), and from the calculations of Buckman et al. (1990) and Rescigno, Elza, \& Lengsfield (1993). The cross sections for electronic excitation into the Rydberg and valence states were obtained from the analytic representation of Shemansky, Ajello, \& Hall (1985) which was based on the experimental measurements of Ajello et al. (1982) and Ajello et al. (1984). The cross sections for the $b^{3} \Sigma_{u}^{+}$state were obtained from the measurements of Nishimura, Danjo \& Sugahara (1985). The cross sections were also measured by Khakoo et al. (1987) and Khakoo \& Segura (1994) and calculated by Baluja, Noble, \& Tennyson (1985), Schneider \& Collins $(1985,1986)$, and Rescigno \& Schneider (1988). The electron impact ionization cross sections were taken from the measurements of Rapp \& Englander-Golden (1965) and Rapp, Englander-Golden, \& Briglia (1965) for the parent 
and dissociative ionizations respectively.

For an average photoelectron energy of $33 \mathrm{eV}$, the mean energy per electronic excitation is about $61 \mathrm{eV}$. Given the total photoelectron energy input in Jupiter of $6.3 \times 10^{10} \mathrm{eV} \mathrm{cm}^{-2} \mathrm{~s}^{-1}$, the total brightness of $\mathrm{H}_{2}$ due to the photoelectron excitation is about $1 \mathrm{kR}$.

\subsection{Ultraviolet Spectra}

The rate of emission from a particular upper rotational level $v^{\prime} J^{\prime}$ of an excited electronic state $i$ of $\mathrm{H}_{2}$ or HD to a lower rotational level $v^{\prime \prime} J^{\prime \prime}$ of the ground electronic state can be written

$$
F\left(i v^{\prime} J^{\prime} \rightarrow v^{\prime \prime} J^{\prime \prime}\right)=\epsilon\left(i v^{\prime} J^{\prime}\right)\left[1-\eta\left(i v^{\prime} J^{\prime}\right)\right] \frac{A_{i v^{\prime} J^{\prime} \rightarrow v^{\prime \prime} J^{\prime \prime}}}{A_{i v^{\prime} J^{\prime}}}
$$

where $\epsilon\left(i v^{\prime} J^{\prime}\right)$ is the rate of excitation of the upper level, $\eta\left(i v^{\prime} J^{\prime}\right)$ is the predissociation efficiency of the upper level, and the third term is the branching ratio for the transition to occur with $A_{i v^{\prime} J^{\prime} \rightarrow v^{\prime \prime} J^{\prime \prime}}$ being the transition probability and $A_{i v^{\prime}} \mathrm{J}^{\prime}$ being the inverse of lifetime of the upper level. The vibrational levels of the high-lying Rydberg states strongly predissociate. The predissociation efficiencies are near unity for the vibrational levels $v^{\prime}>0$ of the $B^{\prime \prime} \Sigma_{u}^{+}$ state, about 0.5 for $v^{\prime}=3-8$ and unity for $v^{\prime}>8$ of the $D^{1} \Pi_{u}$ state, and about 0.4 for $v^{\prime}=1-3$ and unity for $v^{\prime}>3$ of the $D^{\prime 1} \Pi_{u}$ state (Ajello et al. 1984).

Excitation into the $B^{1} \Sigma_{u}^{+}$state occurs also as a result of the cascade $\left(E, F^{1} \Sigma_{g}^{+} \rightarrow B^{1} \Sigma_{u}^{+}\right.$) process following electron impact excitation of the $E, F^{1} \Sigma_{g}^{+}$states. The enhanced rate of excitation of the $B^{1} \Sigma_{u}^{+}$state due to the cascading effects was taken into account according to

$$
\epsilon_{B}^{c}\left(v^{\prime}\right)=\epsilon_{B}\left(v^{\prime}\right)+\sum_{v} q_{v, v^{\prime}} \times \epsilon_{E, F}(v),
$$

where $\epsilon_{B}^{c}\left(v^{\prime}\right)$ and $\epsilon_{B}\left(v^{\prime}\right)$ are the rates of excitation of the $B^{1} \Sigma_{z}^{+}$state with and without the cascade contribution from the $E, F^{1} \Sigma_{g}^{+}$states, $\epsilon_{E, F}(v)$ are the rates of excitation of the $E, F^{1} \Sigma_{g}^{+}$states, and $q_{v, v^{\prime}}$ are the FranckCondon factors for the $E, F^{1} \Sigma_{g}^{+} \rightarrow B^{1} \Sigma_{u}^{+}$transition calculated by Lin (1974).

Radiative transitions from the excited $B^{1} \Sigma_{u}^{+}, B^{\prime 1} \Sigma_{u}^{+}$, $B^{\prime \prime 1} \Sigma_{u}^{+}, C^{1} \Pi_{u}, D^{1} \Pi_{u}$, and $D^{\prime 1} \Pi_{u}$ states of $\mathrm{H}_{2}$ to the ground $X^{1} \Sigma_{g}^{+}$states, and from the excited $B^{1} \Sigma_{u}^{+}$and $C^{1} \Pi_{u}$ states of HD to the ground $X^{1} \Sigma_{g}^{+}$state were included in the calculations of ultraviolet spectra. Radiative transitions from the vibrational levels of the excited $B^{1} \Sigma_{u}^{+}$ and $C^{1} \Pi_{u}$ states of $H_{2}$ and the excited $B^{1} \Sigma_{u}^{+}$state of $H D$ to the vibrational continuum of the ground $X^{1} \Sigma_{g}^{+}$state were included with the transition probabilities calculated by Stephens (1970) and Stephens \& Dalgarno (1972). Radiative transitions from the vibrational levels of the $a^{3} \Sigma_{g}^{+}$ state of $\mathrm{H}_{2}$ to the vibrational continuum of the repulsive $b^{3} \Sigma_{u}^{+}$state were included with the transition probabilities calculated by Kwok et al. (1986). The transition wavelengths were measured by Namioka $(1964,1965)$,
Dabrowski \& Herzberg (1975), and Dabrowski (1984), and calculated by Abgrall et al. (1993a,b).

The emission lines of $\mathrm{H}_{2}$ and $\mathrm{HD}$ terminating at the ground vibrational level of the ground electronic state are optically thick. The photons are reabsorbed and converted predominantly to fluorescence photons in transitions terminating at the excited vibrational levels of the ground electronic state. The basic properties of radiative transfer are different between the solar fluorescence and the photoelectron-excited emission. For the solar fluorescence, absorption of solar photons and subsequent fluorescence emission occur over a range of wavelengths extending into the wings of the line with optical depths being roughly unity. We assumed that the scattered solar photons entering from the top of the atmosphere scatter at approximately the level of unit optical depth at their wavelengths, and that the resulting fluorescence photons connecting to the ground vibrational level traverse approximately unit optical depth to escape the atmosphere with the emission flux attenuated by a factor of $\exp (-\tau)$ which is 0.37 for $\tau=1$. The remaining $63 \%$ of the resonance photons are absorbed and converted mostly to fluorescence lines terminating at higher vibrational levels. Additionally, the fluorescence lines terminating at the excited vibrational level $v=1$ of $\mathrm{H}_{2}$ due to absorption of the solar radiation by the same vibrational level are also optically thick and reabsorbed, and the above treatment of radiative transfer applies. The more elaborate treatment of the radiative transfer presented by Yelle (1988b) should make a minimal difference to the emergent spectrum. This is because the Lyman and Werner band transitions connecting to the ground vibrational level occur at $900-1100 \AA$ where the dominant contribution to the $\mathrm{H}_{2}$ emission come from the transitions originating in the higher-lying excited $B^{\prime 1} \Sigma_{u}^{+}, B^{\prime \prime 1} \Sigma_{u}^{+}, D^{1} \Sigma_{v}^{+}$, and $D^{\prime 1} \Sigma_{u}^{+}$ states.

For the photoelectron-excited emission, on the other hand, the photons are emitted deep in the atmosphere and mostly at wavelengths within several Doppler widths of the line center where the optical depths are the largest. The photons from transitions connecting to the ground vibrational level have to traverse very large optical depths before escaping the atmosphere. In fact most of these photons are absorbed within the atmosphere. We assumed that the photons produced by the photoelectron excitation in transitions to the ground vibrational level are totally absorbed, and that they are converted completely into fluorescence photons in transitions connecting to the excited vibrational levels which escape the atmosphere.

The resulting spectra of $\mathrm{H}_{2}$ and $\mathrm{HD}$ reflect the excitation rates which are dependent on the solar EUV spectrum, on the gas column densities and in detail on the rovibrational populations of the ground electronic states of $\mathrm{H}_{2}$ and $\mathrm{HD}$ which are determined by the temperature. We have calculated the ultraviolet spectra of $\mathrm{H}_{2}$ and $\mathrm{HD}$ for the $\mathrm{H}, \mathrm{H}_{2}$, and $\mathrm{HD}$ column densities of $3 \times 10^{17}, 10^{20}$, and $10^{16} \mathrm{~cm}^{-2}$ respectively, and for the temperature of 
$530 \mathrm{~K}$, characteristic of the Jovian equatorial dayglow, as will be shown in $\S 3.3$. The atomic hydrogen column density was determined from the observations of the Jovian Lyman- $\alpha$ emission profiles (Clarke \& Gladstone 1990; Clarke, Gladstone, \& Ben Jaffel 1991). The $N(\mathrm{H}) / N\left(\mathrm{H}_{2}\right)$ ratio of about $3 \times 10^{-3}$ is appropriate for the observed location of the peak intensity (Strobel et al. 1991). Spectra have also been calculated for the lower temperature of $200 \mathrm{~K}$, typical at the $1 \mu$ bar pressure level, and for the higher temperature of $1000 \mathrm{~K}$, close to the temperatures of $740(+540,-200) \mathrm{K}$ and $800-1200 \mathrm{~K}$ derived from the infrared quadrupole emission of $\mathrm{H}_{2}$ ( $\mathrm{Kim}$ et al. 1990) and the infrared rovibrational emission of $\mathrm{H}_{3}^{+}$(Drossart et al. 1989) respectively. The spectra have been calculated using Gaussian line profiles and have an effective resolution of $3 \AA$ directly comparable to the HUT spectrum.

\subsubsection{Solar Fluorescence Spectra}

\section{(a) $\mathrm{H}_{2}$}

In Figure 3 we present the solar fluorescence spectra of $\mathrm{H}_{2}$ for the $\mathrm{H}, \mathrm{H}_{2}$, and $\mathrm{HD}$ colnmn densities of $3 \times 10^{17}$, $10^{20}$, and $10^{16} \mathrm{~cm}^{-2}$ respectively, and for temperatures of 200,530 , and $1000 \mathrm{~K}$. The brightness of $\mathrm{H}_{2}$ fluorescence increases from $0.96 \mathrm{kR}$ at $200 \mathrm{~K}$ to $1.25 \mathrm{kR}$ at $530 \mathrm{~K}$ and to $1.66 \mathrm{kR}$ at $1000 \mathrm{~K}$ because absorptions from higher rotational levels become more important at higher temperatures. The spectra contain a series of strong emission line features which are produced by strong wavelength coincidences between solar emission lines and absorption lines of $\mathrm{H}_{2}$. Details of the coincidences are listed in Table 1 together with the absorbed fluxes at $530 \mathrm{~K}$, and the resulting strong fluorescence lines of $\mathrm{H}_{2}$ are listed in Table 2 and indicated in Figure 3.

The closest coincidence occurs between the solar Lyman$\beta$ line at $1025.72 \AA$ and the $P(1)$ line of the $(6,0)$ Lyman band of $\mathrm{H}_{2}$ at $1025.93 \AA$ (Feldman \& Fastie 1973). The strong emission features in Figure 3 at $1072 \AA, 1167 \AA$, $1266 \AA, 1366 \AA, 1547 \AA, 1581 \AA, 1608 \AA$, and $1623 \AA$ are the solar Lyman- $\beta$ induced fluorescence of the $P(1)$ lines of the $(6,1),(6,3),(6,5),(6,7),(6,9),(6,11),(6,12)$, $(6,13)$, and $(6,14)$ Lyman bands of $\mathrm{H}_{2}$ respectively. The fluorescence in the $(6,2)$ band contributes significantly to the $1119 \AA$ feature. At $530 \mathrm{~K}$, the solar Lyman- $\beta$ photons induce about $173 \mathrm{R}$ of $\mathrm{H}_{2}$ fluorescence, $59 \%$ of the total solar Lyman- $\beta$ flux of $292 \mathrm{R}$. The solar Lyman- $\beta$ induced fluorescence accounts for $14 \%$ of the total solar fluorescence brightness. The fluorescence becomes weaker with increasing temperature because of the lower population of the rotational level $J=1$ from which the absorption occurs. It reduces from $20 \%$ at $200 \mathrm{~K}$ to $9 \%$ at $1000 \mathrm{~K}$. 19.3 $\mathrm{R}$ or $7 \%$ of the solar Lyman- $\beta$ photons are absorbed by atomic hydrogen followed by $17.5 \mathrm{R}$ of re-emission as Lyman- $\beta$ and $1.8 \mathrm{R}$ of fiuorescence as $H \alpha$ and Lyman$\alpha$. The $17.3 \mathrm{R}$ scattered by $H$ is higher than the value of $10 \mathrm{R}$ calculated by Gladstone (1988) who assumed an atmospheric model with a smaller $H$ column density of $7 \times 10^{16} \mathrm{~cm}^{-2}$. However, higher $\mathrm{H}$ abundances are consistent with the measurements of the Jovian Lyman- $\alpha$ line by Clarke \& Gladstone (1990) and a much broader Lyman$\alpha$ line in the bulge region than appropriate for a $1000 \mathrm{~K}$ Doppler width (Clarke et al. 1991). The $\mathrm{H}_{2}$ fluorescence spectrum is insensitive to the low $\mathrm{H}$ abundance.

A near coincidence occurs between solar Ly $\gamma$ at $972.54 \AA$ and the $R(1)$ line of the $(11,0)$ Lyman band of $\mathrm{H}_{2}$ at $972.63 \AA$. It induces about $69 \mathrm{R}$ of $\mathrm{H}_{2}$ fluorescence, $65 \%$ of the total solar Ly $\gamma$ flux of $106 \mathrm{R}$. The resulting $R(1)+P(3)$ line fluorescence is weaker than the solar $\operatorname{Ly} \beta$ fluorescence due to the lower solar flux at Ly $\gamma$. The solar Ly $\gamma$ fluorescence is most significant in the $(11,14)$ band at $1495 \AA$, and the $(11,7)$ band fluorescence contributes significantly to the $1279 \AA$ feature. $24 \mathrm{R}$ or $22 \%$ of the solar Ly $\gamma$ photons are absorbed by atomic hydrogen followed mostly (17 R) by re-emission as $L y \gamma$ photons and some ( $7 \mathrm{R}$ ) by fluorescence as $\mathrm{Pa} \alpha, \mathrm{H} \alpha, \mathrm{H} \beta, \mathrm{Ly} \alpha$, and $\mathrm{Ly} \beta$.

Strong coincidence also occurs between the solar C II line at $1037.02 \AA$ and $O$ VI line at $1037.61 \AA$ and the $R(1)$ line of the $(5,0)$ Lyman band of $\mathrm{H}_{2}$ at $1037.15 \AA$. Despite the large separation of $0.46 \AA$ between the line centers of the $O$ VI line and the $L(5,0) R(1)$ line, the $O$ VI photons are effectively absorbed by the $\mathrm{H}_{2}$ line because in the line wings the absorption line profile is flat and the optical depth falls very slowly. The optical depth of the $L(5,0) R(1)$ line at wavelength of the center of the $O$ VI line is about 0.5 amounting to about $40 \%$ absorption. The resulting $R(1)+P(3)$ line fluorescence of the $(5,1),(5,3)$, $(5,5),(5,6),(5,8),(5,10)$ and $(5,12)$ bands contributes features at $1084 \AA$ and $1090 \AA, 1181 \AA$ and $1188 \AA, 1283 \AA$ and $1290 \AA, 1334 \AA$ and $1342 \AA, 1436 \AA$ and $1443 \AA$, $1531 \AA$ and $1538 \AA$, and $1608 \AA$ and $1613 \AA$ respectively. Coincidence between the solar N II line at 1085.10 $\AA$ and the $R(4)$ line of the $(2,0)$ Lyman band of $H_{2}$ at $1085.14 \AA$ produces features at $1136 \AA$ and $1151 \AA, 1188 \AA$ and $1204 \AA, 1352 \AA$ and $1370 \AA, 1520 \AA$ and $1538 \AA, 1573 \AA$ and $1590 \AA$, and $1623 \AA$ and $1639 \AA$ due to the $R(4)+P(6)$ line fluorescence of the $(2,1),(2,2),(2,5),(2,8),(2,9)$, and $(2,10)$ bands respectively. The $v^{\prime}=1, J^{\prime}=3$ level of the $C^{1} \Pi_{u}^{-}$state of $\mathrm{H}_{2}$ is excited partly from the ground vibrational level by absorption of the solar N II line at $989.79 \AA$ and partly from the first excited vibrational level by absorption of the solar O VI line at $1031.91 \AA$. The resulting $Q(3)$ line fluorescence of the $(1,1),(1,3),(1,4)$, and $(1,5)$ Werner bands produces the strong features at $1032 \AA, 1119 \AA, 1164 \AA$, and $1209 \AA$ respectively.

Strong Werner band fluorescence of $\mathrm{H}_{2}$ occurs by absorption of the solar Lyman continuum at $870-912 \AA$. The strong broad feature at $1250-1260 \AA$ is produced by the fluorescence in the $(6,10),(7,11),(8,12)$, and $(9,13)$ Werner bands following absorption of the Lyman continuum. The fluorescence in the $(5,10),(6,11),(7,12)$, and $(8,14)$ Werner bands contributes to the relatively weak broad feature at $1277-1283 \AA$.

The solar fluorescence spectrum of $\mathrm{H}_{2}$ depends sensi- 
tively on the temperature because excitations occur from particular rovibrational levels. The strongest temperat ure dependence takes place at $1119 \AA, 1164 \AA$, and $1209 \AA$ at which the emission intensities are enhanced most significantly as the temperature increases. These are produced by the $Q(3)$ line fluorescence in the $(1,3),(1,4)$, and $(1,5)$ Werner bands of $\mathrm{H}_{2}$, which are due partly to excitation from the first excited vibrational level of the ground electronic state. Because of its high excitation energy, the level $v=1$ is populated at a rapidly increasing rate as the temperature increases, and the fluorescence becomes much stronger. Strong temperature dependence occurs also in the spectral shape of the broad feature at $1250-1260 \AA$. The emission is from the vibrational levels $v^{\prime}=6,7,8$, and 9 of the excited $C^{1} \Pi_{v}$ state of $\mathrm{H}_{2}$ populated by absorption of the solar Lyman continuum. Both the increase in the brightness and change in the spectral shape as the temperature increases are the result of more absorption of solar Lyman continunm flux by higher rotational levels. These temperature-sensitive features in the dayglow spectrum can be used to determine the atmospheric temperature. On the other hand, the solar Lyman- $\beta$ induced fluorescence brightness depends less sensitively on the temperature because the absorption occurs from the rotational level $J=1$ whose population is reduced only moderately by increasing temperature.

\section{(b) HD}

The solar fluorescence spectra of $\mathrm{HD}$ are shown in Figure 4 for an HD column density of $10^{16} \mathrm{~cm}^{-2}$ and for the temperatures of 200,530 , and $1000 \mathrm{~K}$. The brightnesses of $H D$ fluorescence are about 42,71 , and $88 \mathrm{R}$ respectively. It would increase by factors of only 1.8 and 2.9 for $\mathrm{HD} / \mathrm{H}_{2}$ abundance ratios of $10^{-3}$ and $10^{-2}$ respectively. Similar to $\mathrm{H}_{2}$, there exist strong wavelength coincidences of solar emission lines and absorption lines of HD. This may provide a specific probe of $\mathrm{HD}$ on Jupiter which because of its low abundance relative to $\mathrm{H}_{2}$ is otherwise likely to be undetectable. The details of line coincidences are listed in Table 1 together with the absorbed flux for each coincidence at $530 \mathrm{~K}$. The resulting strong finorescence lines of $H D$ are indicated in Figure 4 and listed in Table 3 together with the brightness of each fluorescence line.

In analogy to the strong coincidence of the $P(1)$ line of the $(6,0)$ Lyman band of $H_{2}$ and the solar Lyman- $\beta$ line, an exact wavelength coincidence exists between the $R(0)$ line of the $(6,0)$ Lyman band of $H D$ at $1031.91 \AA$ and the strong solar $O$ VI line at $1031.91 A$ which has a solar maximum flux of $6 \mathrm{kR}$ at $1 \mathrm{AU}$ or $218 \mathrm{R}$ at $5.27 \mathrm{AU}$. However, the nature of the absorption is different between HD and $\mathrm{H}_{2}$. For the abundant $\mathrm{H}_{2}$, the optical depth of the absorption line is large and the absorption occurs both in the center region of the line and in a very large portion of the wings of the line. For the much less abundant $\mathrm{HD}$, on the other hand, the absorption line is optically thick only within a few Doppler widths from the line center so that the absorption occurs only in the Doppler core but not in the wings. For an $\mathrm{HD} / \mathrm{H}_{2}$ abundance ratio of $10^{-4}$ or an HD column density of $10^{16} \mathrm{~cm}^{-2}$, the optical depth of the $(6,0) R(0)$ line of $\mathrm{HD}$ is only about 24 at the line center, and it falls below unity at 2 Doppler widths. The optical depth for $\mathrm{HD}$ is small particularly because the absorption is from the rotational level $J=0$ with a lower population than the level $J=1$. Consequently the HD absorbs only $23 \mathrm{R}$ or about $11 \%$ of the solar O VI flux, compared to the $173 \mathrm{R}$ for $\mathrm{H}_{2}$ or $59 \%$ of the solar Lyman- $\beta$ finx. Although the population of the level $J=0$ is lower at higher temperature, more solar $O$ VI photons are absorbed by the broader absorption line. As the result, the absorbed flux increases to $28 \mathrm{R}$ at $1000 \mathrm{~K}$ but decreases to $17 \mathrm{R}$ at $200 \mathrm{~K}$. Even for abnormally high $\mathrm{HD} / \mathrm{H}_{2}$ abundance ratios of $10^{-3}$ and $10^{-2}$, it would be enhanced by factors of only 1.3 and 2.4 respectively. This is because the HD is optically thick only in the Doppler core region where the optical depth falls quickly away from the line center. The strongest fluorescence lines of $\mathrm{HD}$ are the $P(2)$ lines of the $(6,1),(6,3),(6,5),(6,6),(6,8),(6,10),(6,12)$, and $(6,14)$ Lyman bands which are present at $1075 \AA, 1159 \AA$, $1246 \AA, 1290 \AA, 1379 \AA, 1465 \AA, 1545 \AA$, and $1609 \AA$ respectively. The $H D$ fluorescence is substantially reduced owing to the partition between the $R(0)$ and $P(2)$ lines. The strongest fluorescence line of $H D$, the $(6,14) P(2)$ line, has a brightness of $4.8 \mathrm{R}$, an order of magnitude lower than the $49 \mathrm{R}$ for the strongest fluorescence line of $\mathrm{H}_{2}$. The solar fluorescence of $\mathrm{HD}$ becomes $21 \%$ stronger at $1000 \mathrm{~K}$, still much weaker than that of $\mathrm{H}_{2}$. The enhancements would be only 1.3 and 2.4 for $\mathrm{HD} / \mathrm{H}_{2}$ abundance ratios of $10^{-3}$ and $10^{-2}$ respectively.

A weaker line coincidence occurs between the solar N III line at $991.55 \AA$ and the $P(3)$ line of the $(1,0)$ Werner band of $\mathrm{HD}$ at $991.62 \AA$. Its strongest fiuorescence lines are the $(1,4)$ Werner band at $1139 \AA$ and $1143 \AA$. Similar to $\mathrm{H}_{2}$, there is strong Werner band fluorescence of $\mathrm{HD}$ by absorption of the solar Lyman continuum. The strong broad feature at $1257-1267 \AA$ is the fluorescence of $(6,11),(7,12),(8,13)$, and $(9,14)$ Werner bands, and the weaker broad feature at $1284-1289 \AA$ is the fluorescence of $(5,11),(6,12),(7,13),(8,14)$, and $(9,16)$ Werner bands. These solar continuum induced Werner band $f u-$ orescence features of $\mathrm{HD}$ are weaker by an order of magnitude than those of $\mathrm{H}_{2}$.

\subsubsection{Photoelectron Excitation Spectra}

In Figure 5 we present the ultraviolet spectra of $\mathrm{H}_{2}$ due to photoelectron impact excitation for the $\mathrm{H}_{2}$ column density of $10^{20} \mathrm{~cm}^{-2}$ and for temperatures of 200 , 530 , and $1000 \mathrm{~K}$. Large differences exist between the photoelectron-excited spectra and the solar fluorescence spectra. Unlike the solar fluorescence spectra which exhibit a series of bright emission lines, the photoelectronexcited spectra consist of a large number of bands of $\mathbf{H}_{2}$ distributed relatively smoothly with wavelength. The Lyman $\left(B^{1} \Sigma_{u}^{+} \rightarrow X^{1} \Sigma_{g}^{+}\right)$bands are present over the entire 
spectral region, and the Werner $\left(C^{1} \Pi_{u} \rightarrow X^{1} \Sigma_{g}^{+}\right)$bands are concentrated in the wavelength range of $900-1300 \AA$, and the $B^{\prime}, B^{\prime \prime 1} \Sigma_{u}^{+}, D, D^{\prime 1} \Pi_{u} \rightarrow X^{1} \Sigma_{g}^{+}$bands are located at wavelengths shorter than $1200 \AA$. Unlike the solar fluorescence brightness which increases as the temperature increases, the photoelectron excitation brightness is about $0.98 \mathrm{kR}$, independent of the temperature. On the other hand, the distribution of intensity in the photoelectronexcited spectra is significantly altered by the temperature. It tends to be smoother for higher temperatures as the result of the increasing prominence of high rotational lines and the decreasing significance of low rotational lines. The distribution of intensity in the photoelectron-excited spectrum depends less sensitively on the temperature than in the solar fluorescence spectrum because the former is by excitation from all the possible rotational levels of the ground vibrational level while the latter is by selective excitation from particular rotational levels including those of the excited vibrational level $v=1$.

Strong cascading $\left(E, F^{1} \Sigma_{g}^{+} \rightarrow B^{1} \Sigma_{u}^{+}\right)$effects are present in the photoelectron-excited spectra. These effects are also present in the laboratory spectrum produced by impact excitation of $20 \mathrm{eV}$ electrons (Ajello et al. 1982). The cascading effects are the strongest at low electron impact energy because the excitation cross section of the $E, F^{1} \Sigma_{g}^{+}$states is sharply peaked in the threshold region due to the nature of the $X^{1} \Sigma_{g}^{+} \rightarrow E, F^{1} \Sigma_{g}^{+}$transition which is an electric-dipole forbidden transition. The cascade most strongly populates the vibrational level $v^{\prime}=0$ of the $B^{1} \Sigma_{u}^{+}$state, whereas direct excitation populates the levels $v^{\prime}=6,7$. The most intense cascade contributions are the $(0,3)$ vibrational band at $1276 \AA$, the $(0,4)$ band at $1335 \AA$, the $(0,5)$ band at $1395 \AA$, and the $(0,6)$ band at $1455 \AA$, as indicated in Figure 5 . The spectrum is sensitive to the temperature and distinctive differences appear between the low and high temperature spectra as the result of the large spacings between the rotational energy levels of $\mathrm{H}_{2}$. The strong cascade features originate mostly from excitation of low rotational levels, particularly the level $J=1$, so that they are the most significant at low temperatures. As the temperature increases, they become weaker and those of higher $J$ lines become stronger, leading to a smoother spectrum. As illustrated in Figure 5, the cascade spectrum contains the sharpest intensity distribution at $200 \mathrm{~K}$, but the spectral peaks become less significant and the cascade spectra at $530 \mathrm{~K}$ and $1000 \mathrm{~K}$ become smoother. These cascade features in the dayglow spectrum provide a sensitive indicator of the atmospheric temperature.

\subsubsection{Synthetic Spectra}

In Figure 6 we present synthetic spectra containing contributions from the solar fluorescence of $\mathrm{H}_{2}$ and $\mathrm{HD}$ and from the photoelectron excitation of $\mathrm{H}_{2}$. The synthetic spectra contain a series of bright emission features originating from the solar fluorescence of $\mathrm{H}_{2}$ superimposed on the relatively smooth spectra due to the photoelectron excitation of $\mathrm{H}_{2}$. The solar fluorescence contributes $1.32 \mathrm{kR}$, including $1.25 \mathrm{kR}$ from $\mathrm{H}_{2}$ and $0.07 \mathrm{kR}$ from HD, and accounts for $57 \%$ of the total $2.3 \mathrm{kR}$ of the Jovian dayglow emission, while the photoelectron excitation contributes $0.98 \mathrm{kR}$ and accounts for the remaining $43 \%$. The distribution of intensity in the synthetic spectrum is sensitive to the temperature especially for the emission lines excited from the first excited vibrational level and from high rotational levels. The intensities of the strong features at $1032 \AA, 1119 \AA, 1164 \AA$, and $1209 \AA$ which are due partly to excitation from the excited vibrational level are the most sensitive to the temperature. They increase by about $40 \%$ for a temperature increasing from $530 \mathrm{~K}$ to $1000 \mathrm{~K}$, and decrease by a factor of 1.3 for a temperature decreasing from $530 \mathrm{~K}$ to $200 \mathrm{~K}$. The strengths of the strong features at $1276 \AA, 1335 \AA, 1395 \AA$, and $1455 \AA$ which are due mostly to cascading decrease by about $15 \%$ for a temperature increasing from $530 \mathrm{~K}$ to $1000 \mathrm{~K}$ and increase by about $15 \%$ for a temperature decreasing from $530 \mathrm{~K}$ to $200 \mathrm{~K}$. Inclusion of the HD fluorescence affects the synthetic spectra only slightly for an $\mathrm{HD} / \mathrm{H}_{2}$ abundance ratio of $10^{-4}$ and a temperature of $530 \mathrm{~K}$. Even for much higher abundance ratios and temperatures, the $\mathrm{HD}$ fluorescence would be only moderately stronger.

\section{Jovian Dayglow}

\subsection{Spectrum}

In Figure 7, the synthetic spectrum is compared with the Jovian equatorial dayglow spectrum presented from Feldman et al. (1993). The adopted $\mathrm{H}, \mathrm{H}_{2}$, and $\mathrm{HD}$ column densities are $3 \times 10^{17}, 10^{20}$, and $10^{16} \mathrm{~cm}^{-2}$ respectively, and the temperature is $530 \mathrm{~K}$. The solar fluorescence spectrum of $\mathrm{HD}$ is included in the figure. The strong fluorescence lines and the strong cascade features of $\mathrm{H}_{2}$ and the Lyman- $\alpha,-\beta$, and $-\gamma$ lines of $\mathrm{H}$ are indicated in the figure. The spectra are compared only in the spectral region between $900 \AA$ and $1500 \AA$ because at wavelengths longer than $1500 \AA$ solar reflection due to the large planetary albedo becomes the dominant contributor to the observed signal (Feldman et al. 1993). There is a good agreement between the synthetic spectrum and the dayglow spectrum. In addition, all the strong $\mathrm{H}_{2}$ features observed between $1500 \AA$ and $1500 \AA$ are reproduced by the synthetic spectrum.

Strong fluorescence of $\mathrm{H}_{2}$ induced by the solar Lyman$\beta$ is present in the dayglow spectrum. The line features at $1072 \AA, 1167 \AA, 1266 \AA, 1366 \AA$, and $1462 \AA$ have been identified by Feldman et al. (1993) to be the $P(1)$ lines of the $(6,1),(6,3),(6,5),(6,7)$, and $(6,9)$ Lyman bands respectively. In addition, a large part of the $1119 \AA$ feature is due to the $(6,2) P(1)$ line fluorescence. The very good agreement between the synthetic spectrum and the dayglow spectrum allows a reliable determination of the solar Lyman- $\beta$ fiuorescence brightness. It is $173 \mathrm{R}$ which 
is $73 \%$ higher than the estimate of Feldman et al. (1993). The strong feature at $1495 \AA$ is identified to be mostly the solar Lyman- $\gamma$ fluorescence $R(1)+P(3)$ lines of the $(11,14)$ Lyman band of $H_{2}$. The very good agreement between the synthetic spectrum and the dayglow spectrum allows a reliable determination of the solar Lyman- $\gamma$ fluorescence brightness to be $69 \mathrm{R}$. The strong features at $1119 \AA$ and $1164 \AA$ are identified as the $Q(3)$ line fluorescence of the $(1,3)$ and $(1,4)$ Werner bands which is due partly to excitation by the solar N II line from the ground vibrational level and partly to excitation by the solar $O$ VI line from the first excited vibrational level. Most of the Lyman- $\beta$ red wing at $1032 \AA$ is identified to be the $Q(3)$ line fluorescence in the $(1,1)$ Werner band. Strong $H_{2}$ emission by absorption of the solar Lyman continuum is clearly seen at $1250-1260 \AA$ and $1277-1283 \AA$.

There is a strong emission feature at $1026 \AA$ in the dayglow spectrum. It consists of two components: $17.5 \mathrm{R}$ from the solar Lyman- $\beta$ resonance scattering by atomic hydrogen and 10.4 $\mathrm{R}$ from the solar Lyman $-\beta$ induced fluorescence of $\mathrm{H}_{2}$. The photoelectron impact excitation of $\mathrm{H}_{2}$ contributes very little because of the strong selfabsorption. It follows that the total brightness of the $1026 \mathrm{~A}$ emission is about $28 \mathrm{R}$, in good agreement with the $30 \pm 7 \mathrm{R}$ estimated by Feldman et al. (1993). It follows also that the total solar Lyman- $\beta$ induced fluorescence is $192 \mathrm{R}$, including $173 \mathrm{R}$ of $\mathrm{H}_{2}$ fluorescence and about $19 \mathrm{R}$ of $H$ fluorescence. The latter contains about $2 R$ of fluorescence as $\mathrm{H} \alpha$ and Lyman- $\alpha$. They are about $66 \%$ of the solar Lyman- $\beta$ photons, and the rest is absorbed by the $\mathrm{CH}_{4}$ at the homopause. There is also a strong emission at $973 \AA$ due to the solar Lyman- $\gamma$ resonance scattering by atomic hydrogen with a brightness of $16 \pm 5 \mathrm{R}$ (Feldman et al. 1993), in agreement with the value of $17 \mathrm{R}$ predicted by our calculations. The agreement suggests that the intensity and profile of the solar Lyman- $\beta$ and Lyman- $\gamma$ lines used in our calculations are adequate.

Although the solar fluorescence and photoelectron excitation model is very successful in reproducing the dayglow spectrum, there erist some strong observed features not accounted for by the model. Among the most significant is the strong emission feature at $1302 \AA$ where the $\mathrm{H}_{2}$ emission is very weak. This $\mathrm{O} I$ feature is likely an artifact since it has a strange and narrow shape and it should be broad at the HUT resolution since it is a triplet. There is some terrestrial contamination by the O I triplet together with some in the Io torus spectrum that was subtracted. Other significant features in the dayglow spectrum but not present in the synthetic spectrum are located at $1201 \AA, 1357 \AA, 1405 \AA$, and $1419 \AA$. Possible contamination by the Io torus emission are likely because there are very strong features in the torns spectrum at these wavelengths. All the above features probably do not originate in Jupiter. Althongh the strong features at $1175 \AA, 1229 \AA$, and $1236 \AA$ in the dayglow spectrum are significantly stronger than predicted, their derived intensities are uncertain due to the influence of the grating-scattered Lyman- $\alpha$ (Feldman et al. 1993). The only unidentified strong feature in the dayglow spectrum is the broad feature centered at $1475 \AA$. This feature can not be accounted for by simply adjusting the temperature and must originate from other species.

\subsection{Absorption by $\mathrm{CH}_{4}$}

There are several strong features of $\mathrm{B}_{2}$ at short wavelengths predicted in the synthetic spectrum which are significantly weaker in the measured spectrum. They include the features at $1054 \AA$ due to the $(0,1)$ Werner band excited by photoelectron impact, at $1072 \AA$ due to the $(6,1)$ Lyman band produced by the solar Lyman- $\beta$ fluorescence, at $1084 A$ and $1090 \AA$ due to the $(5,1)$ Lyman band partly from the solar $C$ II and $O$ VI line fluorescence, and at $1120 \AA$ due to the $(1,3)$ Werner band and the $(3,1)$ Lyman band excited by photoelectron impact and to the $(1,3)$ and $(6,2)$ Werner bands produced by the solar $O V I$ and Lyman- $\beta$ fluorescence respectively. The largest disagreement occurs in the $1054 \AA$ feature which may result from our neglect of self-absorption by $\mathrm{H}_{2}$ from the first excited vibrational level. Self-absorption in $v=1$ may be important because photoelectron excitation occurs deep in the atmosphere. This could be clarified by a more elaborate treatment of radiative transfer. The other strong features are emission lines either connecting to higher excited vibrational levels of the ground electronic state or produced by solar fluorescence, and they are not affected by self-absorption by the vibrationally excited $\mathrm{H}_{2}$.

The weakness of these features in the dayglow spectrum may be the result of absorption by the $\mathrm{CH}_{4}$ at the homopause. The photoionization of $\mathrm{H}_{2}$ occurs deep in the atmosphere, above or within the regions where $\mathrm{CH}_{4}$ is abundant. The solar fluorescence of $\mathrm{H}_{2}$ is mostly by absorption in the wings of the $\mathrm{H}_{2}$ lines which also takes place deep in the atmosphere. In addition to the self-absorption by the $\mathrm{H}_{2}$ at the ground vibrational level which was taken into account in our calculations, the photoelectron-excited emission of $\mathrm{H}_{2}$ and part of the solar fluorescence of $\mathrm{H}_{2}$ may be subject to absorption by $\mathrm{CH}_{4}$. The $\mathrm{CH}_{4}$ absorption may be strong at wavelengths shorter than $1300 \AA$ where the absorption cross section of $\mathrm{CH}_{4}$ increases rapidly (Okabe \& Becker 1963). Comparison of the synthetic and dayglow spectra suggests a line-of-sight attenuation factor of less than 0.6 , corresponding to an effective $\mathrm{CH}_{4}$ optical depth of at most 0.5 . It is smaller than the value of 0.63 found for the Jovian auroral regions (Trafton et al. 1994).

\section{3. $\quad \mathrm{H}_{2}$ Abundance and Temperature}

The $\mathrm{H}_{2}$ column density and the atmospheric temperature at the peak of the dayglow emission can be determined by minimizing the chi-square function defined as

$$
\chi^{2}(T, N)=\frac{1}{n} \sum_{i=1}^{n} \frac{\left(F_{i}^{s}(T, N)-F_{i}^{d}\right)^{2}}{F_{i}^{z}(T, N)+F_{i}^{d}}
$$


where $n$ is the number of spectral data points which is 1106, and $F_{i}^{s}$ and $F_{i}^{d}$ denote the fluxes of the synthetic and dayglow spectra respectively. The spectral regions where the strong and broad atomic hydrogen Lyman- $\alpha$, $-\beta$ and $-\gamma$ are present were excluded in calculating $\chi^{2}$. The $\chi^{2}$ depends on the $\mathrm{H}_{2}$ column density $N$ and the temperature $T$. The $\chi^{2}$ curves are shown as a function of $N$ for $T=530 \mathrm{~K}$ in Figure $8 \mathrm{a}$ and as a function of $T$ for $N=10^{20} \mathrm{~cm}^{-2}$ in Figure $8 \mathrm{~b}$. The $\chi^{2}$ is sensitive to the $\mathrm{H}_{2}$ column density and has a well determined minimum at $10^{20} \mathrm{~cm}^{-2}$. The minimum depends on the magnitude of the solar flux, and the derived $\mathrm{H}_{2}$ column density would be higher for a lower solar flux than used in our calculations. The derived $\mathrm{H}_{2}$ column density of $10^{20} \mathrm{~cm}^{-2}$ is in very good agreement with that given by a Jovian atmospheric model based on auroral observations of Jupiter (Trafton et al. 1994). This suggests that the solar EUV spectrum used in our calculations is adequate. The $\chi^{2}$ is less sensitive to the temperature especially in the neighborhood of the minimum at $530 \mathrm{~K}$, and the atmospheric temperature at the peak of the dayglow emission is likely to be near $530 \mathrm{~K}$. This temperature balances best the low significance of the cascade contribution and the low contribution by absorption from the vibrationally excited $\mathrm{H}_{2}$. The former requires a high temperature and the latter a low temperature. The less well determined temperature may in part reflect the presence of a significant temperature distribution even in the peak emitting region, including possible variations in the $\mathrm{H}_{2}$ temperature with either altitude or position. The uncertainty of the solar spectrum may also contribute because the derived temperature depends on the shape of the solar spectrum, especially on the relative strengths of the strong lines and the shape of the Lyman continuum. The derived atmospheric temperature of $530 \mathrm{~K}$ places the peak emission of $\mathrm{H}_{2}$ above or within the homopause, consistent with moderate absorption of the $\mathrm{H}_{2}$ emission by the $\mathrm{CH}_{4}$ at the homopause of Jupiter. It falls into the temperature range from $400-450 \mathrm{~K}$ to $700-750 \mathrm{~K}$ (Clarke et al. 1994) and of $530 \pm 100 \mathrm{~K}$ (Trafton et al. 1994) derived from auroral spectra of Jupiter. Stellar occultation measurements indicate a much lower mean atmospheric temperature of $176 \pm 12 \mathrm{~K}$ at a higher pressure level below the homopanse (Hubbard et al. 1995). This low temperature is likely the result of efficient cooling by the $\mathrm{CH}_{4}$ on Jupiter. Our results imply that there is a large temperature gradient just above the $\mathrm{CH}_{4}$ layer. As the $\mathrm{CH}_{4}$ abundance decreases above the homopause, the temperature increases drastically from roughly $200 \mathrm{~K}$ to $500-600 \mathrm{~K}$.

\section{Discussion}

\subsection{Contribution of Solar Fluorescence}

Our calculations show that solar fluorescence contributes $1.32 \mathrm{kR}$ to and accounts for $57 \%$ of the total $2.3 \mathrm{kR}$ of the Jovian dayglow emission, consistent with the earlier suggestion by Yelle (1988a) that solar fluorescence is the major excitation source. Feldman et al. (1993) identified solar Lyman- $\beta$ induced fluorescent lines of $\mathrm{H}_{2}$ in the dayglow spectrum and estimated the contribution to be 100 R. From the calculations of Yelle (1988a), which showed that Lyman- $\beta$ induced fluorescence is responsible for $20 \%-25 \%$ of the total fluorescence, Feldman et al. (1993) concluded that solar fluorescence produces $400-500 \mathrm{R}$ of dayglow, equal to $17 \%-22 \%$ of the observed $2.3 \mathrm{kR}$. Their estimate of $100 \mathrm{R}$ for the contribution from the solar Lyman- $\beta$ fluorescence is lower than our value of $173 \mathrm{R}$. Their value was derived from a visual comparison of the dayglow spectrum with the laboratory spectrum of electron impact excitation (Ajello et al. 1984) which was taken for an electron energy of $100 \mathrm{eV}$ and room temperature. A direct comparison of the dayglow and laboratory spectra is hampered because the dayglow emission is produced by slower photoelectrons which have an average energy of $33 \mathrm{eV}$ exciting hotter $\mathrm{H}_{2}$ at a temperature of $530 \mathrm{~K}$. The electron impact excitation spectrum is sensitive to the electron energy and the gas temperature. The comparison is limited also because secondary electrons are dominant in the dayglow but do not contribute to the laboratory spectrum. Further, the low counting statistics of the observational data are responsible for a large uncertainty in deriving the underlying electron impact contribution to the dayglow spectrum. We believe also that Feldman et al. (1993) have overestimated the relative contribution of solar Lyman- $\beta$ induced fiuorescence to the total solar fluorescence. The contribution depends strongly on the temperature. The value of $20 \%-25 \%$ is appropriate to a temperature of $200 \mathrm{~K}$. For the higher temperature of $530 \mathrm{~K}$ in the Jovian upper atmosphere, the contribution is reduced to $14 \%$. A combination of these two factors results in an underestimate by a factor of $2.5-3.1$ for the relative contribution of solar fluorescence to the Jovian dayglow.

\subsection{Contribution of Photoelectron Excitation}

With the solar fluorescence contributing $1.32 \mathrm{kR}$ to and accounting for $57 \%$ of the observed Jovian dayglow emission, and with the remaining $0.98 \mathrm{kR}$ or $43 \%$ supplied by the photoelectron excitation, the solar fluorescence and photoelectron impact excitation can account for the Jovian dayglow emission without any additional energy source. By underestimating the contribution of solar fluorescence to the dayglow emission, Feldman et al. (1993) were led to overestimate the contribution of electron impact excitation. They inferred a value of $1.8-1.9 \mathrm{kR}$ or $78 \%-83 \%$ of the observed dayglow emission. Because this is about twice the energy source expected from the photoelectrons, Feldman et al. (1993) argued for an additional energy source.

Feldman et al. (1993) also argued for an additional energy source from a consideration of the energy spectrum 
of the exciting electrons. Pointing to the basic overall similarity between the equatorial dayglow spectrum and the south polar auroral spectrum, they claimed that the energies of the exciting electrons for the dayglow and for the aurora should be similar, suggesting that the additional electrons exciting the dayglow must be more energetic than the low energy photoelectrons. However, the overall similarity, if it exists, is probably not significant, given the severe contamination of the auroral spectrum by strong terrestrial features (Feldman et al. 1993). The overall similarity more likely occurs because energetic electrons produce slower secondary electrons and they are the dominant source of excitation. We note that there do occur significant differences between the dayglow and auroral spectra in the relative strengths of the $\mathrm{H}_{2}$ rotational lines.

Feldman et al. (1993) pointed to an additional indication of energetic electron excitation: the apparent absence in the dayglow spectrum of an appreciable cascade contribution to the Lyman band emission from higher-lying $E$ and $F$ states in the spectral region between $1300 \AA$ and $1450 \mathrm{~A}$. Ajello et al. (1982) had drawn attention to the strong cascading effects seen in a laboratory spectrum with $20 \mathrm{eV}$ electrons compared to laboratory spectra taken with 100 and $300 \mathrm{eV}$ electrons. However the postulated energetic electrons would produce a substantial number of slower secondary electrons which will contribute strongly to the cascade. The apparent contradiction can be resolved by recognizing the sensitivity to the temperature of the cascade intensity distribution. The peaks of emission due to the cascading are excited from the rotational level $J=1$. At low temperatures these peaks are the strongest with the sharpest intensity distribution. As the temperature increases the peaks become weaker, and the weaker features excited from higher rotational levels become stronger, so that the cascade spectrum becomes smoother and the peaks become less significant. The smoothness of the cascade spectrum in the dayglow spectrum is a good indication of high temperatures. The close agreement between the dayglow spectrum and the $530 \mathrm{~K}$ synthetic spectrum in Figure 7 confirms that cascading effects are present in the dayglow spectrum. Feldman et al. (1993) remarked that the cascade contribution appears to be absent from both the HUT auroral spectrum and the IUE auroral spectrum of Waite et al. (1988). Since auroral emission arises from energetic electron excitation and is expected to originate in hotter regions, cascading effects are less significant in the auroral spectrum.

\subsection{Solar Fluorescence and Photoelectron Excitation}

The relative contributions of solar fluorescence and photoelectron excitation depend on the solar flux. Solar fluorescence is proportional to the solar fiux between $900 \AA$ and $1100 \AA$ and photoelectron excitation is de- termined by the solar flux at wavelengths shorter than $500 \mathrm{~A}$. Our adopted solar maximum intensities of Lyman$\beta$ and $\mathrm{He} \mathrm{II}(304 \mathrm{~A})$ lines of $8.1 \mathrm{kR}$ and $7.6 \mathrm{kR}$ at $1 \mathrm{AU}$ respectively are based on a reliable solar irradiance measurement and model and our factor of 1.9 for the solar cycle variation of Lyman- $\beta$ is very close to the value of 2 advocated by Lean (1987). Our fluxes of Lyman- $\beta$ and He II lines should be more secure than the estimates of $6 \mathrm{kR}$ of Strobel et al. (1991) and of $12 \mathrm{kR}$ of Torr et al. (1979) respectively, but even such an unlikely large decrease from $57 \%$ to $40 \%$ in the fluorescence contribution could be compensated by a comparable increase from $43 \%$ to $60 \%$ in the photoelectron contribution, without compromising the agreement between theory and observation within their uncertainties. We conclude that the dayglow spectrum can be attributed to a combination of solar fluorescence and photoelectron excitation and no additional energy source need be invoked.

\subsection{Detectability of HD}

The exact coincidence of the strong solar $O$ VI line and the $R(0)$ line of the $(6,0)$ Lyman band of HD and the absorption of the solar Lyman continunm by HD produce a strong HD fluorescence. The HD fluorescence lines should be sought in the spectral regions where the $\mathrm{HD}$ emission is the strongest and the $\mathrm{H}_{2}$ emission is the weakest. The predicted $\mathrm{HD} / \mathrm{H}_{2} \mathrm{flux}$ ratios are highest at $1075 \AA$, $1159 \AA, 1379 \AA, 1465 \AA, 1545 \AA$, and $1607 \AA$. At these wavelengths, there are indeed significant emission features in excess of the $\mathrm{H}_{2}$ features in the dayglow spectrum. However these excessive intensities are too high to be accounted for by HD for the assumed HD abundance: The order of $10^{17} \sim 10^{18} \mathrm{~cm}^{-2}$ of HD would be required were the excess emissions from $\mathrm{HD}$. It may be possible that more HD fluorescence could be produced deeper in the atmosphere than the $\mathrm{H}_{2}$ emission. However this may not be statistically significant given the poor counting statistics.

With higher spectral resolution and sensitivity, it may be possible to separate the HD fluorescence lines from the dominant $\mathrm{H}_{2}$ spectrum. Search with the Hubble Space Telescope which has a much higher spectral resolution and sensitivity than the HUT may be successful. In Figure 9 we present the $\mathrm{HD} / \mathrm{H}_{2}$ flux ratio as a function of the wavelength at the high resolution of $0.3 \AA$ which can be achieved with the $B S T / G H R S$. The $\mathrm{H}_{2}$ and $H D$ column densities are assumed to be $10^{20} \mathrm{~cm}^{-2}$ and $10^{16} \mathrm{~cm}^{-2} \mathrm{re}$ spectively. The highest ratios are 6.4 at $1607 \AA, 4.3$ at $1379 \AA, 4.1$ at $1159 \AA$, and 3.0 at $1465 \AA$. In Figure 10 we show the predicted $0.3 A$ resolution spectra of $\mathrm{H}_{2}$ and $\mathrm{HD}$ in which some strongest $\mathrm{HD}$ lines are well separated from the nearby strong $H_{2}$ lines. This may allow a detection of $\mathrm{HD}$ in the Jovian atmosphere. The best candidates for the HD detection are the $P(2)$ lines of the $(6,8)$ and $(6,10)$ Lyman bands of $H D$ located at $1379 \AA$ and $1465 \AA$ respectively. The $R(0)$ line of the $(6,14)$ Lyman band of $\mathrm{HD}$ at 
$1607 \AA$ is very strong but it may be dominated by solar refiection. Although the $P(2)$ line of the $(6,2)$ Lyman band of $\mathrm{HD}$ is strong and well separated from the nearly strong $\mathrm{H}_{2}$ lines, it is partially absorbed by the $\mathrm{CH}_{4}$ in the Jovian atmosphere. Some of the strongest HD lines overlap in wavelength with $\mathrm{H}_{2}$ lines but have comparable line strengths. The $P(2)$ line of the $(6,14)$ Lyman band of $H D$ at $1609 \AA$ is the strongest $\mathrm{HD}$ line. It has a brightness of about $60 \%$ of that of the overlapping $\mathrm{H}_{2}$ features which is contributed mainly by the $P(3)$ line of the $(6,13)$ Lyman band of $\mathrm{H}_{2}$. Similar cases are the $P(2)$ lines of the $(6,12)$ and $(6,1)$ Lyman bands of $H D$ at $1545 \AA$ and $1075 \AA$ respectively. Again solar reflection may dominate these $\mathrm{HD}$ lines at $1609 \AA$ and $1545 \AA$ and absorption of the HD line at $1075 \AA$ by the $\mathrm{CH}_{4}$ may be significant.

It should be noted that the solar fluorescence spectrum of HD depends on the magnitude and shape of the solar spectrum and on the details of the absorption by $\mathrm{H}_{2}$ of the solar radiation. The interpretation of the observations would be enhanced by a further refinement in our knowledge of the solar EUV spectrum on which the predicted spectra of $\mathrm{H}_{2}$ and $\mathrm{HD}$ depend.

This work was supported by the National Aeronautics and Space Administration, under grant NAGW-1561. We are greatly indebted to Prof. P. D. Feldman who offered helpful comments and suggestions and provided us with the numerical values of the observational data of the Jovian equatorial dayglow presented in Feldman et al. (1993), which we reproduce in Figure 7. Helpful comments from Dr. R. V. Yelle are acknowledged. We are also indebted to Dr. R. Gredel who provided us with his compilation of electron impact excitation cross sections of $\mathrm{H}_{2}$.

\section{REFERENCES}

Abgrall, H., Roueff, E. Launay, F., Roncin, J.-Y., \& Subtil, J.-L. 1993a, A\&AS, 101, 273

Abgrall, H., Roueff, E. Launay, F., Roncin, J.-Y., \& Subtil, J.-L. 1993b, A\&AS, 101, 323

Ajello, J. M., Shemansky, D., Kwok, T. L., \& Yung, Y. L. 1984, Phys. Rev. A, 29, 636

Ajello, J. M., Srivastava, S. K., \& Yung, Y. L. 1982, Phys. Rev. A, 25, 2485

Allison A. C., \& Dalgarno, A. 1970, Atomic Data, 1, 289

Backx, C., Wight, G. R., \& Van der Wiel, M. J. 1976, J. Phys. B, 9, 315

Baluja, K. L., Noble, C. J., \& Tennyson, J. 1985, J. Phys. $B, 18, L 851$

Brunger, M. J., Buckman, S. J., Newan, D. S., \& Alle, D. T. 1991 J. Phys. B, 24, 1435

Buckman, S. J., Bruger, M. M., Newman, D. S., Schitchler, G., Alston, S., Norcross, D. W., Morrision, M. A., Saha, B. C., Danby, G., \& Trail, W. K. 1990, Phys. Rev. Lett., 65, 3253

Cacelli, I., Moccia, R., \& Rizzo, A. 1993, J. Chem. Phys., 98,8742

Chung, Y. M., Lee, E.-M., Masuoka, T., \& Samson, J. A. R. 1993, J. Chem. Phys., 99, 885

Clarke, J. T., \& Gladstone, G. R. 1990, J. Geophys. Res., 95, 21281

Clarke, J. T., Gladstone, G. R., \& Ben Jaffel, L. 1991, Geophys. Res. Lett., 18, 1935

Clarke, J. T., Hudson, M., \& Yung, Y. L. 1987, J. Geophys. Res., 92, 15139

Clarke, J. T., Jaffel, L. B., Vidal-Madjar, A., Gladstone, G. R., Waite, J. H., Jr., Prangé, R., Gérard, J.-C., \& Ajello, J. M. 1994, ApJ, 430, L73

Cravens, T. E., Victor, G. A., \& Dalgarno, A., 1975, Planet. Space. Sci., 23, 1059

Dabrowski, I. 1984, Can. J. Phys., 62, 1639

Dabrowski, I., \& Herzberg, G. 1975, Can. J. Phys., 54, 525

Drossart, P., et al. 1989, Nature, 340, 539

Feldman, P. D., \& Fastie, W. G. 1973, ApJ, 185, L101

Feldman, P. D., McGrath, M. A., Moos, H. W., Durrance, S. T., Strobel, D. F., \& Davidsen, A. F. 1993, ApJ, 406, 279

Gladstone, G. R. 1988, J. Geophys. Res., 93, 14630

Glass-Manjean, M. 1984, Atomic Data and Nuclear Data Tables, 30, 301

Gredel, R., Lepp, S., \& Dalgarno, A., 1987, ApJ, 323, L137 
Gredel, R., Lepp, S., Dalgarno, A., \& Herbst, E. 1989, ApJ, 347, 289

Hinteregger, H. E., Pukui, K., \& Gilson, B. R. 1981, Geophys. Res. Lett., 8, 1147

Hoegy, W. R., Pesnell, W. D., Woods, T. N., \& Rottman, G. J. 1993, Geophys. Res. Lett., 13, 1335

Hubbard, W. B., Haemmerle, V., Porco, C. C., Rieke, G. H., \& Rieke, M. J. 1995, ICARUS, 113, 103

Hudson, M. K., Clarke, J. T., \& Warren, J. A. 1989, J. Geophys. Res., 94, 6517

Karzas, W. J., \& Latter, R. 1961, ApJS, 6, 167

Khakoo, M. A., \& Segura, J. 1994, J.Phys.B, 27, 2355

Khakoo, M. A., Trajmar, S., McAdams, R., \& Shyn, T. W. 1987, Phys. Rev. A, 35, 2832

Kim, S. J., Drossart, P., Caldwell, J., \& Maillard, J. P. 1990, Icarus, 84, 54

Kwok, T. L., Dalgarno, A., \& Posen, A. 1985, Phys. Rev. A, 32, 646

Kwok, T. L., Guberman, S., Dalgarno, A., \& Posen, A. 1986, Phys. Rev. A, 34, 1962

Lean, J. 1987, J. Geophys. Res., 92, 839

Lemaire, P., Charra, J., Jouchoux, A., Vidal-Madjar, A., Artzner, G., E., Vial, J. C., Bonnet, R. M., \& Skumanich, A. 1978, ApJ, 223, L55

Lin, C. S. 1974, J. Chem. Phys., 60, 4660

McGrath, M. A., Feldman, P. D., Ballester, G. E., \& Moos H. W. 1989, Geophys. Res. Lett., 16, 583

Namioka, T. 1964, J. Chem. Phys., 41, 2141

Namioka, T. 1965, J. Chem. Phys., 43, 1636

Nishimura, H., \& Danjo, A. 1986, J. Phys. Soc. Japan, 55,3031

Nishimura, H., Danjo, A., \& Sugahara, H. 1985, J. Phys. Soc. Japan, 54, 1757

Okabe, H., \& Becker, D. A. 1963, J. Chem. Phys., 39, 2549

Rapp, D., \& Englander-Golden, P. 1965, J. Chem. Phys., 43,1

Rapp, D., Englander-Golden, P., \& Briglia, D. D. 1965, J. Chem. Phys., 42, 4081

Rescigno, T. N., Elza, B. K., \& Lengsfield, B. H. 1993, J. Phys. B, 26, L567

Rescigno, T. N., \& Schneider, B. I. 1988, J. Phys. B, 21, L691

Schmidtke, G., Woods, T. N., Worden, J., Rottman, G. J., Doll, H., Wita, C., \& Solomon, S. C . 1992, Geophys. Res. Lett., 19, 2175

Schneider, B. I., \& Collins, L. A. 1985, J. Phys. B, 18, L57
Schneider, B. I., \& Collins, L. A. 1986, Phys. Rev. A, 33, 2982

Shemansky, D. E. 1985, J. Geophys. Res., 90, 2673

Shemansky, D. E., \& Ajello, J. M. 1983, J. Geophys. Res., 88,459

Shemansky, D. E., Ajello, J. M., \& Hall, D. T. 1985, ApJ, 296, 765

Stephens, T. L. 1970, PhD Thesis, Harvard University

Stephens, T. L., \& Dalgarno, A. 1972, J. Quant. Spectrose. Rad. Transf., 12, 569

Strobel, D. F., Yelle, R. V., Shemansky, D. E., \& Atreya, S. K. 1991, in Uranus, ed. J. J. Bergstrahl, E. D. Miner, \& M. S. Matthews (Tucson: Univ. Arizona Press), 65

Tobiska, W. K. 1991, J. Atmos. Terr. Phys., 53, 1005

Tobiska, W. K. 1993, J. Geophys. Res., 98, 18879

Torr, M. R., Torr, D. G., Ong, R. A., \& Hinteregger, H. E. 1979, Geophys. Res. Lett., 6, 771

Tousey, R. Purcell, J. D., Austin, W. E., Garrett, D. L., \& Widing, K. G. 1964, Space Research, 4, 703

Trafton, L. M., Gérard, J. C., Munhoven, G., \& Waite, J. H., Jr. 1994, ApJ, 421, 816

Waite, J. H., Jr., Clarke, J. T., Cravens, T. E., \& Hammond, C. M. 1988, J. Geophys. Res., 93, 7244

Yelle, R. V. 1988a, Geophys. Res. Lett., 15, 1145

Yelle, R. V. 1988b, ApJ, 332, 514

Yelle, R. V., MeConnell, J. C., Sandel, B. R., \& Broadfoot, A. L. 1987, J. Geophys. Res., 92, 15110

This 2-column preprint was prepared with the AAS IATEX macros v3.0. 


\section{FIGURE CAPTIONS}

Fig. 1.- Solar EUV spectra at 5.27 AU for the high, moderate, and low solar activities indicated by the values of the solar $10.7 \mathrm{~cm}$ index. They consist of numerous solar emission lines superimposed on the He I continuum between $450 \AA$ and $504 \AA$, the $\mathrm{H}$ Lyman continuum between $700 \AA$ and $912 \AA$, and the C I continuum between $913 \AA$ and $1100 \AA$. The continuum fluxes are presented by integrating over a $1 \AA$ bin. The total integrated fluxes between $18 \AA$ and $500 \AA$ and between $800 \AA$ and $1200 \AA$ are indicated.

Fig. 2.- The flux of photoelectrons produced by the solar radiation in the Jovian upper atmosphere for the high solar activity appropriate for the HUT observations of Jupiter. The column density of $\mathrm{H}_{2}$ is $10^{20} \mathrm{~cm}^{-2}$.

Fig. 3.- The solar fluorescence spectra of $\mathrm{H}_{2}$ for the $\mathrm{H}_{2}$ column density of $10^{20} \mathrm{~cm}^{-2}$ and for the temperatures of 200,530 , and $1000 \mathrm{~K}$. The strong fluorescence lines due to coincidence of strong solar emission lines and absorption lines of $\mathrm{H}_{2}$ are indicated.

Fig. 4.- The solar fluorescence spectra of $H D$ for the HD column density of $10^{16} \mathrm{~cm}^{-2}$ and for the temperatures of 200,530 , and $1000 \mathrm{~K}$. The strong fluorescence lines due to coincidence of strong solar emission lines and absorption lines of $\mathrm{HD}$ are indicated.

Fig. 5.- The photoelectron impact excitation spectra of $\mathrm{H}_{2}$ for the $\mathrm{H}_{2}$ column density of $10^{20} \mathrm{~cm}^{-2}$ and for the temperatures of 200,530 , and $1000 \mathrm{~K}$. The strong emission lines due to the cascading effects are indicated.

Fig. 6.- The synthetic spectra of $\mathrm{H}_{2}$ and $\mathrm{HD}$ for the $\mathrm{H}_{2}$ and $\mathrm{HD}$ column densities of $10^{20} \mathrm{~cm}^{-2}$ and $10^{16} \mathrm{~cm}^{-2}$ respectively, and for the temperatures of 200,530 , and $1000 \mathrm{~K}$.
Fig. 7.- The Jovian equatorial dayglow spectrum observed by the HUT in comparison with the theoretical synthetic spectrum. The HD spectrum (the lower solid curve) is included for comparison. The $\mathrm{H}, \mathrm{H}_{2}$, and $\mathrm{HD}$ column densities are $3 \times 10^{17} \mathrm{~cm}^{-2}, 10^{20} \mathrm{~cm}^{-2}$, and $10^{16} \mathrm{~cm}^{-2}$ respectively, and the temperature is $530 \mathrm{~K}$. The strong solar fluorescence lines and the strong cascade features of $\mathrm{H}_{2}$ identified in the dayglow spectrum are indicated, together with the atomic hydrogen Lyman- $\alpha,-\beta$, and $-\gamma$ lines.

Fig. 8.- The $\chi^{2}$ calculated in comparison of the synthetic spectrum and the Jovian equatorial dayglow spectrum. (a) Dependence on the $\mathrm{H}_{2}$ column density is shown at the temperature of $530 \mathrm{~K}$. (b) Dependence on the atmospheric temperature is shown for the $\mathrm{H}_{2}$ column density of $10^{20} \mathrm{~cm}^{-2}$. The minimum of the $\chi^{2}$ corresponds to the $\mathrm{H}_{2}$ column density of $10^{20} \mathrm{~cm}^{-2}$ and the temperature of $530 \mathrm{~K}$.

Fig. 9.- The $\mathrm{HD} / \mathrm{H}_{2}$ flux ratios at the high resolution of $0.3 \AA$ for the $\mathrm{H}_{2}$ and $\mathrm{HD}$ column densities of $10^{20} \mathrm{~cm}^{-2}$ and $10^{16} \mathrm{~cm}^{-2}$ respectively, and for the temperature of $530 \mathrm{~K}$.

Fig. 10.- The high resolution $(0.3 \AA)$ spectra of $\mathrm{H}_{2}$ and $\mathrm{HD}$ for the $\mathrm{H}_{2}$ and $\mathrm{HD}$ column densities of $10^{20} \mathrm{~cm}^{-2}$ and $10^{16} \mathrm{~cm}^{-2}$ respectively, and for the temperature of $530 \mathrm{~K}$. Strong $\mathrm{HD}$ lines are indicated. The strong $\mathrm{HD}$ lines well separated from the $\mathrm{H}_{2}$ spectrum serve as candidates for a possible detection of $\mathrm{HD}$ on Jupiter. 
TABLE 1

Coincidences of Solar Emission Lines and Absorption Lines of $\mathrm{H}_{2}$ and $\mathrm{HD}$

\begin{tabular}{|c|c|c|c|c|c|c|c|c|}
\hline \multicolumn{3}{|c|}{ Solar Emission Lines } & \multicolumn{6}{|c|}{ Absorption Lines of $\mathrm{H}_{2}$ and $\mathrm{HD}$} \\
\hline \multirow[t]{2}{*}{ Species } & \multirow{2}{*}{$\begin{array}{l}\text { Wavelength } \\
(\AA)\end{array}$} & \multirow{2}{*}{$\begin{array}{r}\text { Flux } \\
\text { (R) }\end{array}$} & \multirow[t]{2}{*}{ Molecule } & \multicolumn{2}{|c|}{ Transition } & \multirow{2}{*}{$\begin{array}{l}\text { Wavelength } \\
(\AA)\end{array}$} & \multicolumn{2}{|c|}{ Absorption } \\
\hline & & & & Band & Line & & (R) & $(\%)$ \\
\hline$\overline{\mathrm{H}} \mathrm{Ly}-\gamma$ & 972.54 & 106 & $\mathrm{H}_{2}$ & $\mathrm{~L}(11,0)$ & $\bar{R}(1)$ & 972.63 & $\frac{1-1}{69}$ & $\frac{65}{65}$ \\
\hline $\mathrm{N}$ II & 989.79 & 24 & $\mathrm{H}_{2}$ & $\mathrm{~W}(1,0)$ & $Q(3)$ & 989.73 & 21 & 88 \\
\hline N III & 991.55 & 48 & HD & $\mathrm{W}(1,0)$ & $P(3)$ & 991.62 & 2.6 & 5 \\
\hline В Ly- $\beta$ & 1025.72 & 292 & $\mathrm{H}_{2}$ & $\mathrm{~L}(6,0)$ & $P(1)$ & 1025.93 & 173 & 59 \\
\hline O VI & 1031.91 & 218 & $\mathrm{H}_{2}$ & $W(1,1)$ & $Q(3)$ & 1031.86 & 12 & 6 \\
\hline $\mathrm{O}$ VI & 1031.91 & 218 & $\mathrm{HD}$ & $\mathrm{L}(6,0)$ & $R(0)$ & 1031.91 & 23 & 11 \\
\hline C II & 1037.02 & 43 & $\mathrm{H}_{2}$ & $\mathrm{~L}(5,0)$ & $R(1)$ & 1037.15 & 43 & 100 \\
\hline O VI & 1037.61 & 162 & $\mathrm{H}_{2}$ & $\mathrm{~L}(5,0)$ & $R(1)$ & 1037.15 & 64 & 40 \\
\hline N II & 1085.10 & 58 & $\mathrm{H}_{2}$ & $L(2,0)$ & $R(4)$ & 1085.14 & 36 & 62 \\
\hline
\end{tabular}


TABLE 2

Solar Fluorescence Lines of $\mathrm{H} 2$

\begin{tabular}{|c|c|c|c|c|c|}
\hline Source & $\begin{array}{c}\text { Absorption } \\
\text { (R) }\end{array}$ & Band & $\overline{\text { Line }}$ & $\begin{array}{c}\text { Wavelength } \\
(\AA)\end{array}$ & $\begin{array}{c}\text { Brightness } \\
(\mathrm{R})\end{array}$ \\
\hline \multirow[t]{11}{*}{$\overline{\mathrm{H}} \mathrm{Ly}-\beta$} & 173 & $\mathrm{~L}(6,0)$ & $P(1)$ & 1025.93 & 10.4 \\
\hline & & $\mathrm{L}(6,1)$ & & 1071.62 & 9.4 \\
\hline & & $\mathrm{L}(6,2)$ & & 1118.61 & 6.1 \\
\hline & & $\mathrm{L}(6,3)$ & & 1166.76 & 14.3 \\
\hline & & $\mathrm{L}(6,5)$ & & 1265.68 & 15.7 \\
\hline & & $\mathrm{L}(6,7)$ & & 1365.66 & 14.9 \\
\hline & & $\mathrm{L}(6,9)$ & & 1461.97 & 14.6 \\
\hline & & $\mathrm{L}(6,11)$ & & 1546.72 & 15.0 \\
\hline & & $\mathrm{L}(6,12)$ & & 1581.11 & 10.5 \\
\hline & & $\mathrm{L}(6,13)$ & & 1607.50 & 48.7 \\
\hline & & $\mathrm{L}(6,14)$ & & 1623.37 & 5.6 \\
\hline \multirow[t]{7}{*}{ H Ly- $\gamma$} & 69 & $\mathrm{~L}(11,2)$ & $R(1)+P(3)$ & $1055.54,1061.46$ & 4.0 \\
\hline & & $\mathrm{L}(11,3)$ & & $1098.31,1104.38$ & 2.7 \\
\hline & & $\mathrm{L}(11,5)$ & & $1185.53,1191.80$ & 3.9 \\
\hline & & $\mathrm{L}(11,7)$ & & $1272.81,1279.10$ & 4.6 \\
\hline & & $\mathrm{L}(11,9)$ & & $1356.07,1362.04$ & 3.1 \\
\hline & & $\mathrm{L}(11,10)$ & & $1394.24,1399.87$ & 2.6 \\
\hline & & $\mathrm{L}(11,14)$ & & $1493.83,1495.56$ & 7.4 \\
\hline \multirow[t]{8}{*}{ C II, O VI } & 107 & $\mathrm{~L}(5,0)$ & $R(1)+P(3)$ & $1037.15,1043.50$ & 5.6 \\
\hline & & $\mathrm{L}(5,1)$ & & $1083.86,1090.45$ & 10.6 \\
\hline & & $\mathrm{L}(5,3)$ & & $1181.29,1188.32$ & 11.2 \\
\hline & & $\mathrm{L}(5,5)$ & & $1282.79,1290.14$ & 7.0 \\
\hline & & $\mathrm{L}(5,6)$ & & $1334.28,1341.72$ & 5.9 \\
\hline & & $\mathrm{L}(5,8)$ & & $1436.09,1443.46$ & 9.1 \\
\hline & & $\mathrm{L}(5,10)$ & & $1530.74,1537.52$ & 10.8 \\
\hline & & $\mathrm{L}(5,12)$ & & $1607.90,1613.18$ & 35.5 \\
\hline \multirow[t]{6}{*}{$\mathrm{N}$ II } & 36 & $\mathrm{~L}(2,1)$ & $R(4)+P(6)$ & $1135.70,1151.16$ & 5.1 \\
\hline & & $\mathrm{L}(2,2)$ & & $1187.89,1203.92$ & 5.3 \\
\hline & & $\mathrm{L}(2,5)$ & & $1352.32,1369.71$ & 4.8 \\
\hline & & $\mathrm{L}(2,8)$ & & $1520.12,1537.56$ & 6.0 \\
\hline & & $\mathrm{L}(2,9)$ & & $1573.40,1590.29$ & 7.6 \\
\hline & & $\mathrm{L}(2,10)$ & & $1623.36,1639.23$ & 2.7 \\
\hline \multirow[t]{4}{*}{ N II, O VI } & 34 & $\mathrm{~W}(1,1)$ & $Q(3)$ & 1031.86 & 4.9 \\
\hline & & $\mathrm{W}(1,3)$ & & 1119.08 & 8.9 \\
\hline & & $\mathrm{W}(1,4)$ & & 1163.80 & 11.8 \\
\hline & & $W(1,5)$ & & 1208.93 & 5.1 \\
\hline \multirow[t]{4}{*}{ H Ly-Continuum } & & $W(6,10)$ & & $1250-1260$ & \\
\hline & & $W(7,11)$ & & & \\
\hline & & $W(8,12)$ & & & \\
\hline & & $W(9,13)$ & & & \\
\hline \multirow[t]{4}{*}{ H Ly-Continuum } & & $W(5,10)$ & & $1277-1283$ & \\
\hline & & $W(6,11)$ & & & \\
\hline & & $W(7,12)$ & & & \\
\hline & & $\mathrm{W}(8,14)$ & & & \\
\hline
\end{tabular}


TABLE 3

Solar Fluorescence Lines of HD

\begin{tabular}{|c|c|c|c|c|c|}
\hline Source & $\begin{array}{c}\text { Absorption } \\
(\mathrm{R})\end{array}$ & Band & Line & $\begin{array}{c}\text { Wavelength } \\
(\AA)\end{array}$ & $\begin{array}{l}\text { Brightness } \\
\text { (R) }\end{array}$ \\
\hline \multirow[t]{9}{*}{$\mathrm{OVI}$} & 23 & $\mathrm{~L}(6,0)$ & $R(0)+P(2)$ & $1031.91,1034.76$ & 1.05 \\
\hline & & $\mathrm{L}(6,1)$ & & $1072.10,1075.04$ & 2.07 \\
\hline & & $\mathrm{L}(6,3)$ & & $1155.54,1158.66$ & 2.07 \\
\hline & & $\mathrm{L}(6,5)$ & & $1242.37,1245.63$ & 1.21 \\
\hline & & $\mathrm{L}(6,6)$ & & $1286.63,1289.95$ & 1.15 \\
\hline & & $\mathrm{L}(6,8)$ & & $1375.52,1378.88$ & 1.74 \\
\hline & & $\mathrm{L}(6,10)$ & & $1462.14,1465.42$ & 1.94 \\
\hline & & $\mathrm{L}(6,12)$ & & $1541.62,1544.61$ & 2.13 \\
\hline & & $\mathrm{L}(6,14)$ & & $1606.59,1608.97$ & 7.15 \\
\hline \multirow[t]{4}{*}{ N III } & 2.6 & $W(1,1)$ & $R(1)+P(3)$ & $1023.97,1028.43$ & 0.53 \\
\hline & & $W(1,3)$ & & $1099.73,1104.42$ & 0.36 \\
\hline & & $W(1,4)$ & & $1138.61,1143.41$ & 0.78 \\
\hline & & $W(1,5)$ & & $1177.99,1182.86$ & 0.51 \\
\hline \multirow[t]{4}{*}{ H Ly-Continuum } & & $W(6,11)$ & & $1257-1267$ & \\
\hline & & $W(7,12)$ & & & \\
\hline & & $W(8,13)$ & & & \\
\hline & & $W(9,14)$ & & & \\
\hline \multirow[t]{5}{*}{ H Ly-Continuum } & & $W(5,11)$ & & $1283-1287$ & \\
\hline & & $W(6,12)$ & & & \\
\hline & & $W(7,13)$ & & & \\
\hline & & $W(8,14)$ & & & \\
\hline & & $W(9,16)$ & & & \\
\hline
\end{tabular}




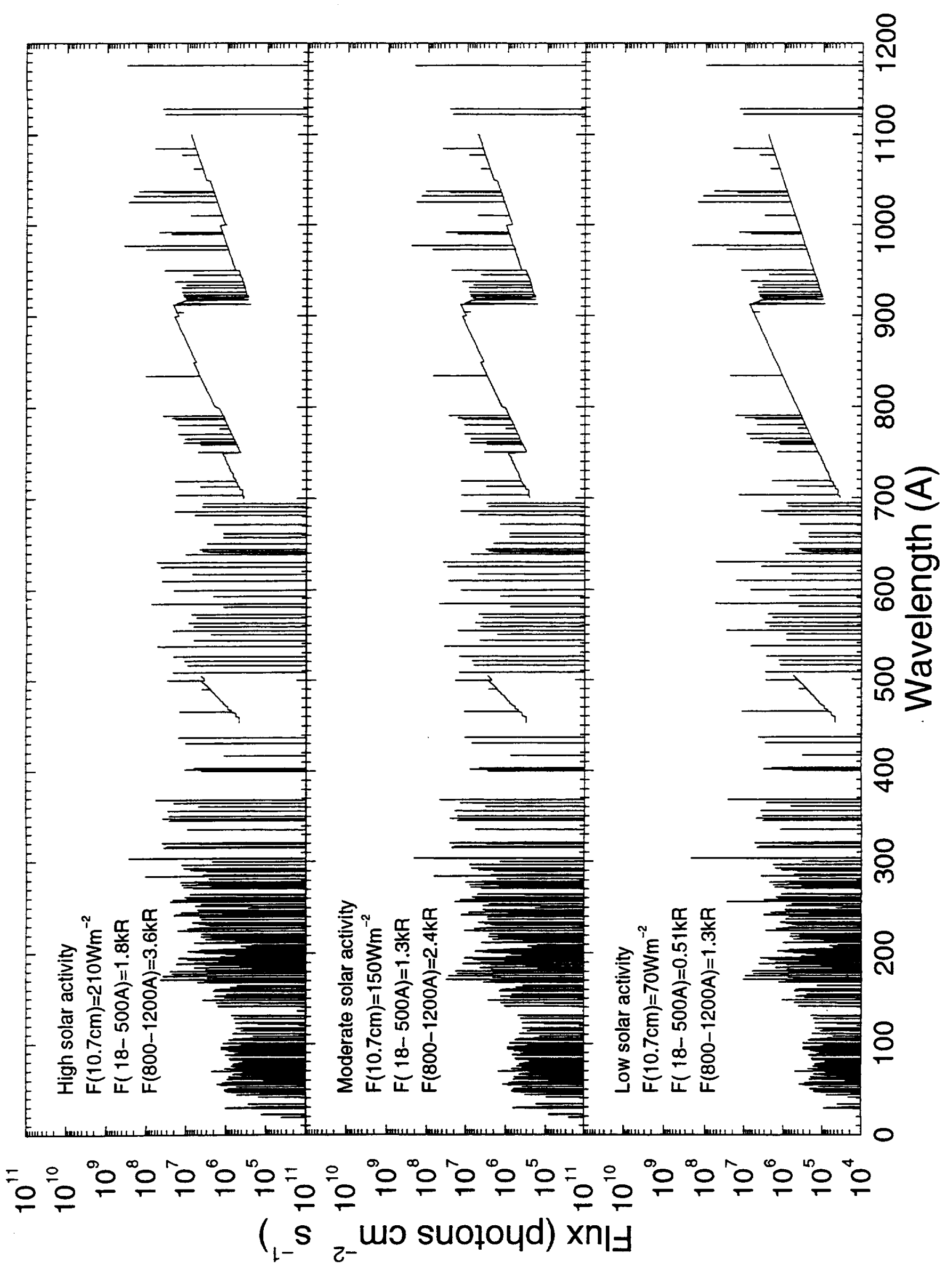




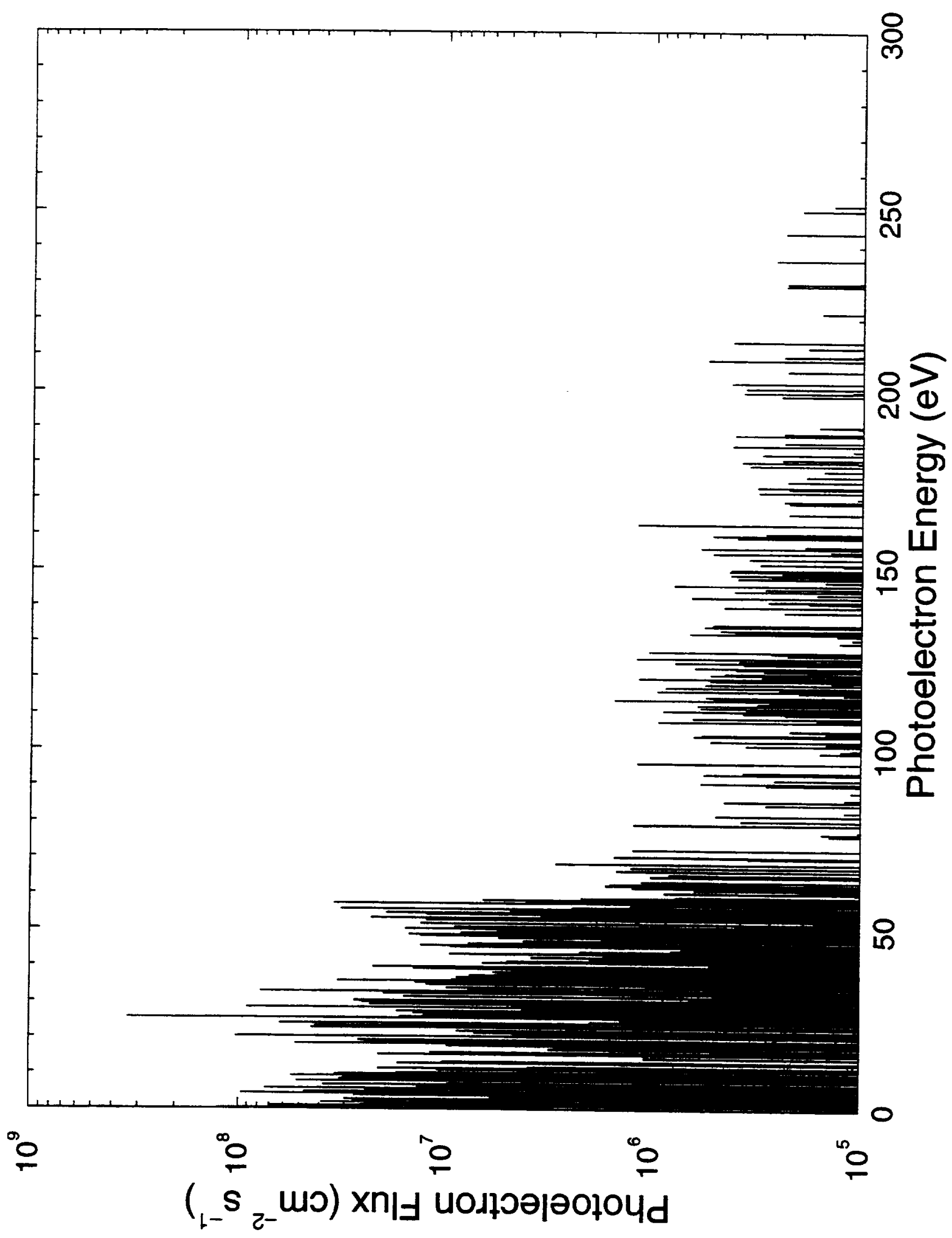




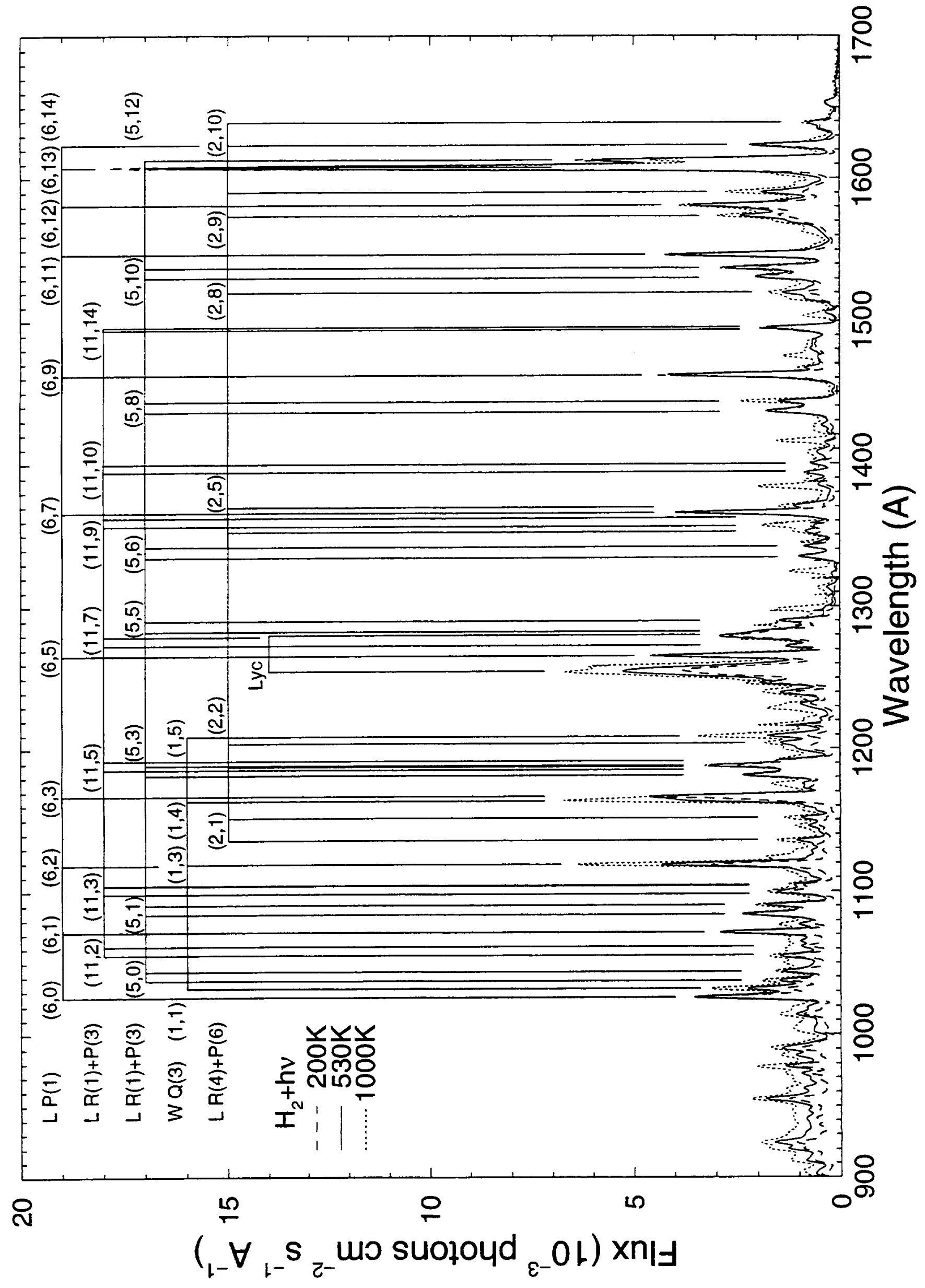




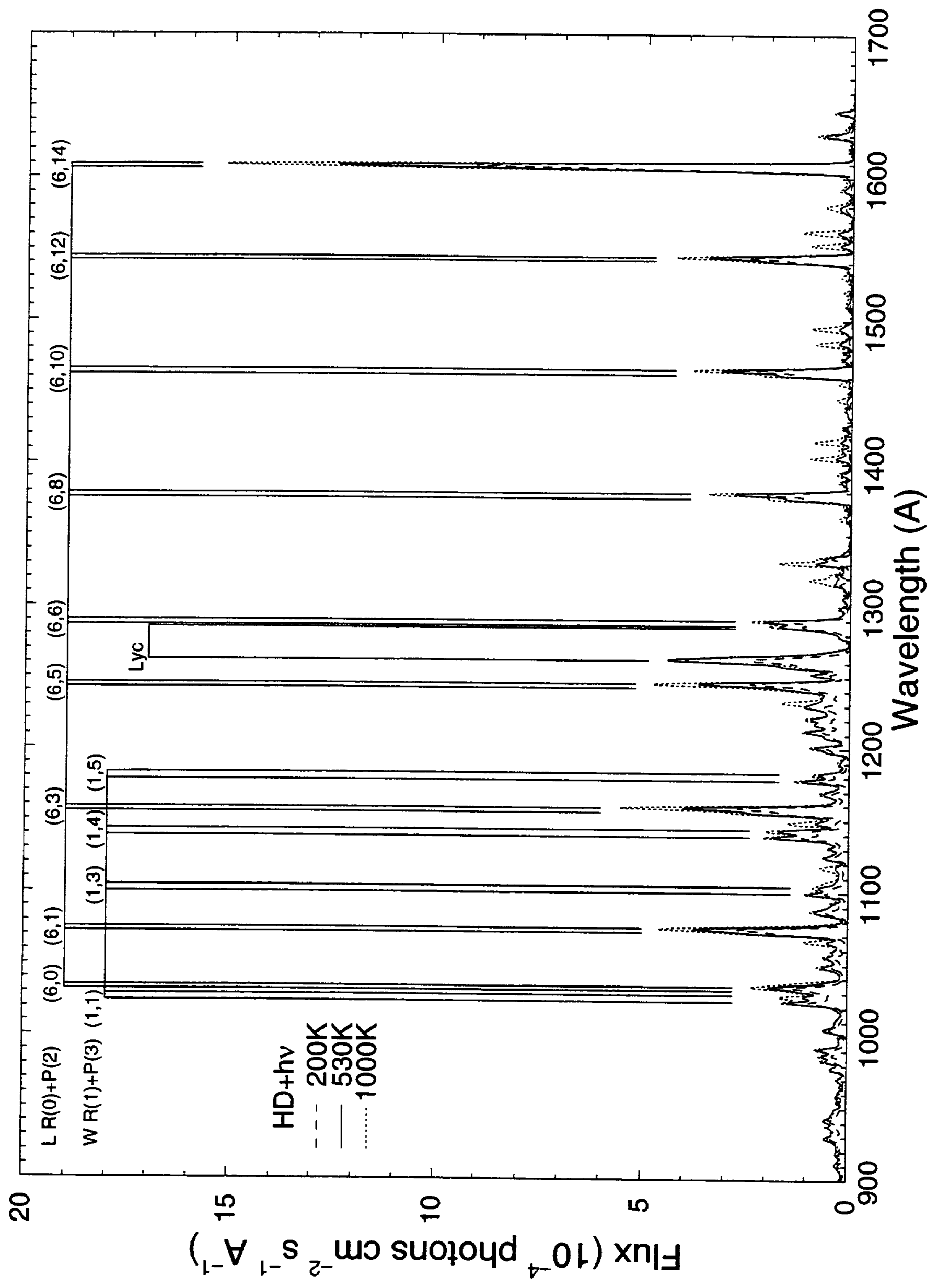




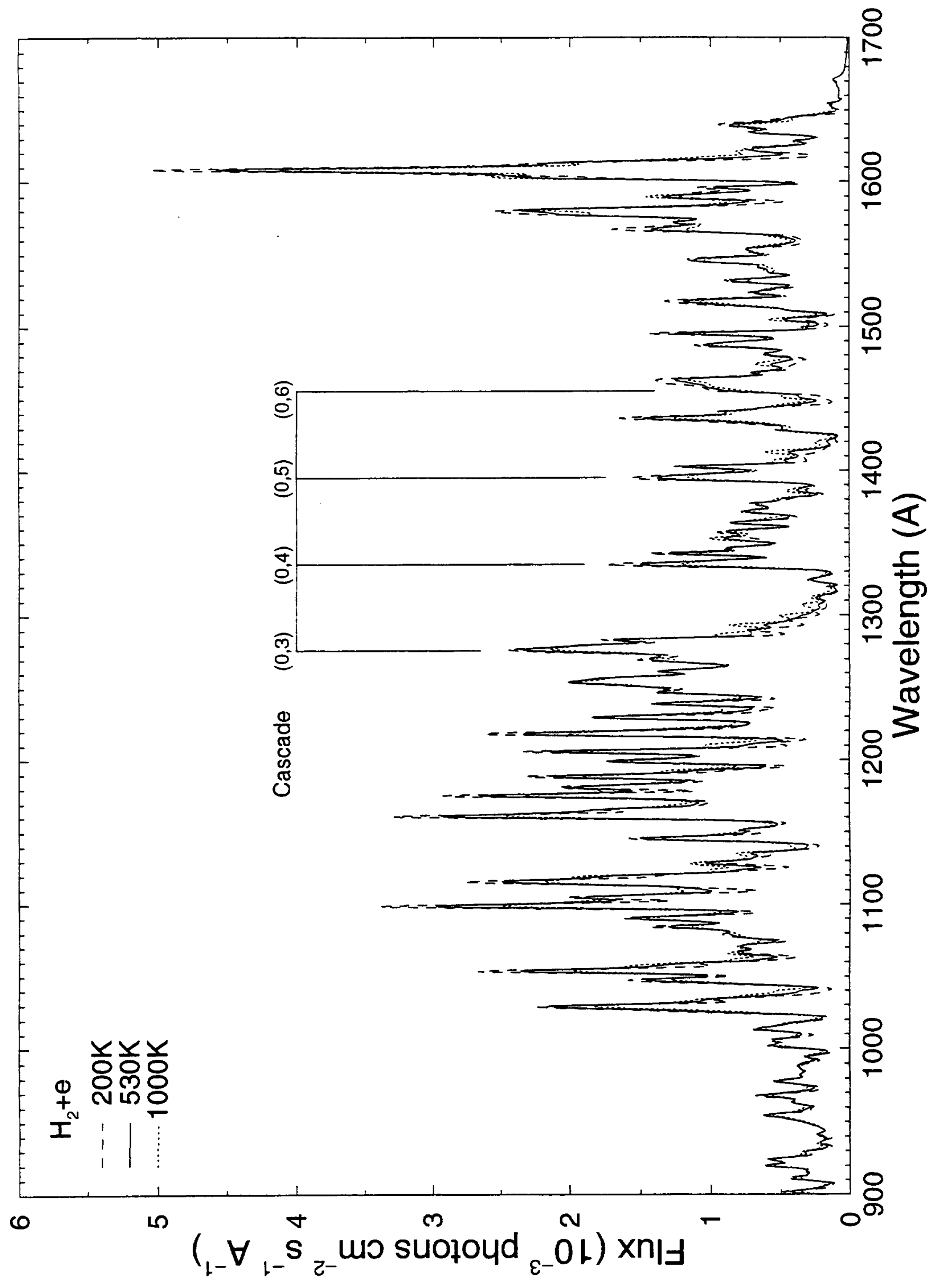




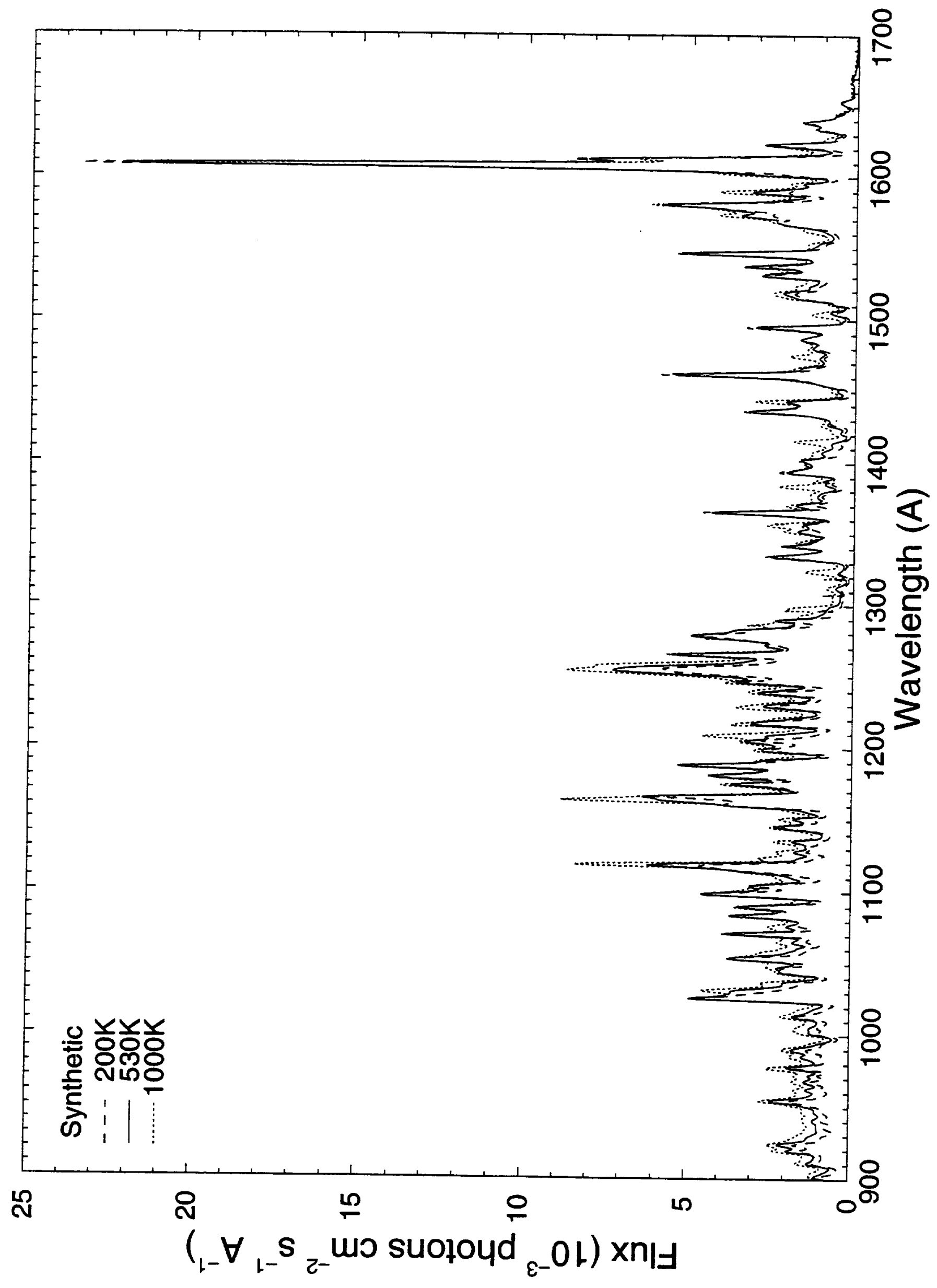

0
0
$\frac{0}{3}$
욤 


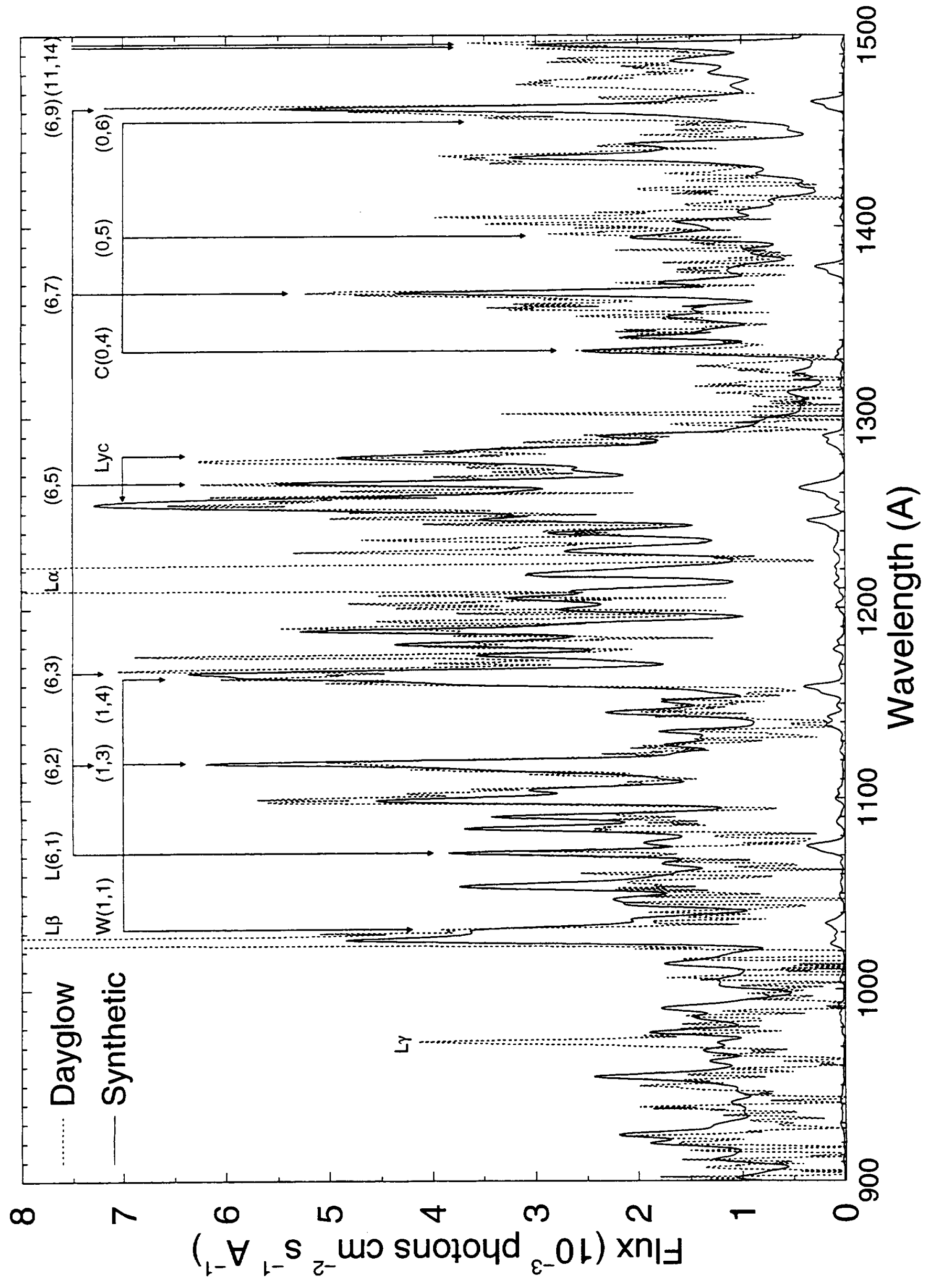




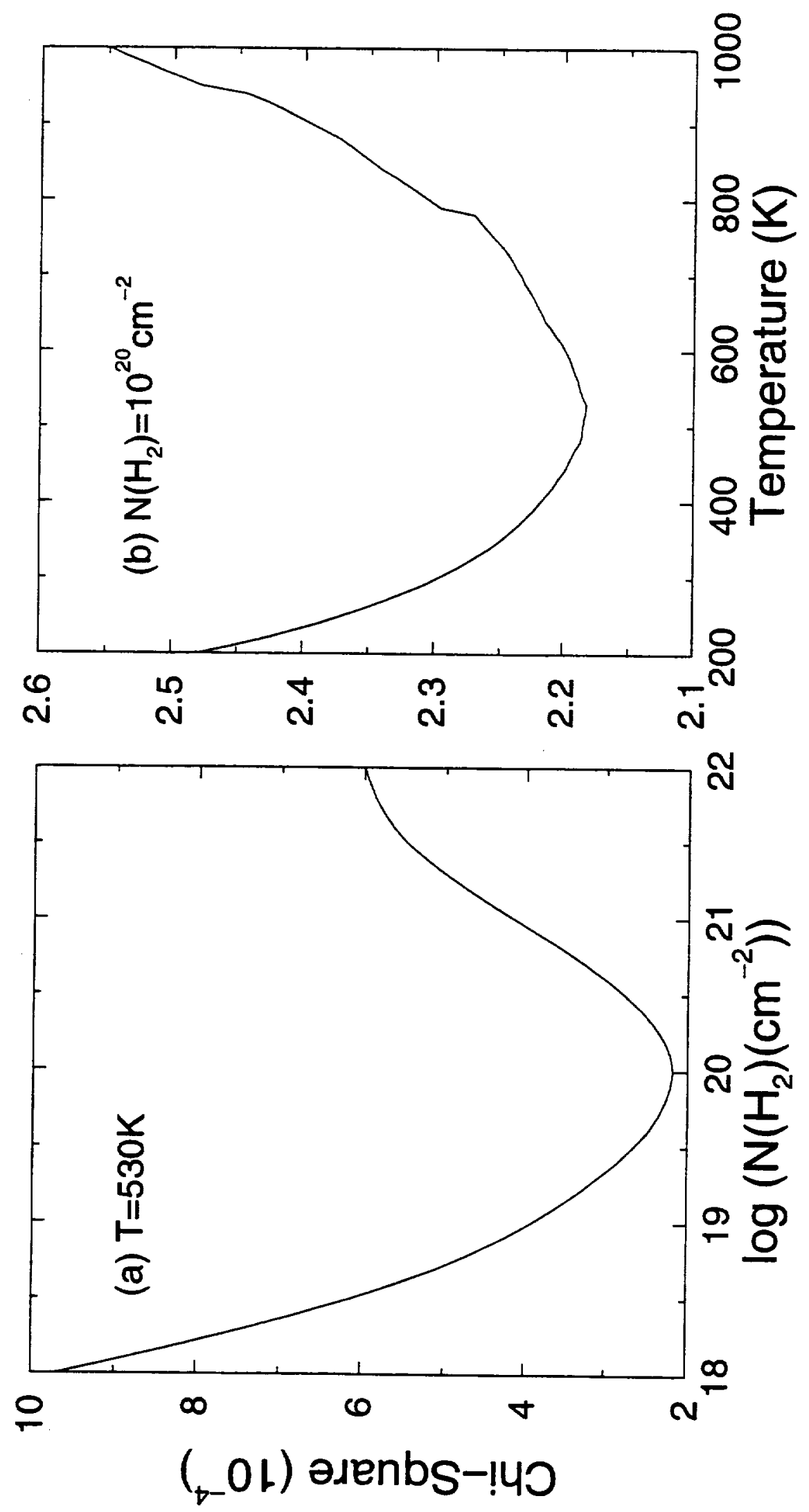




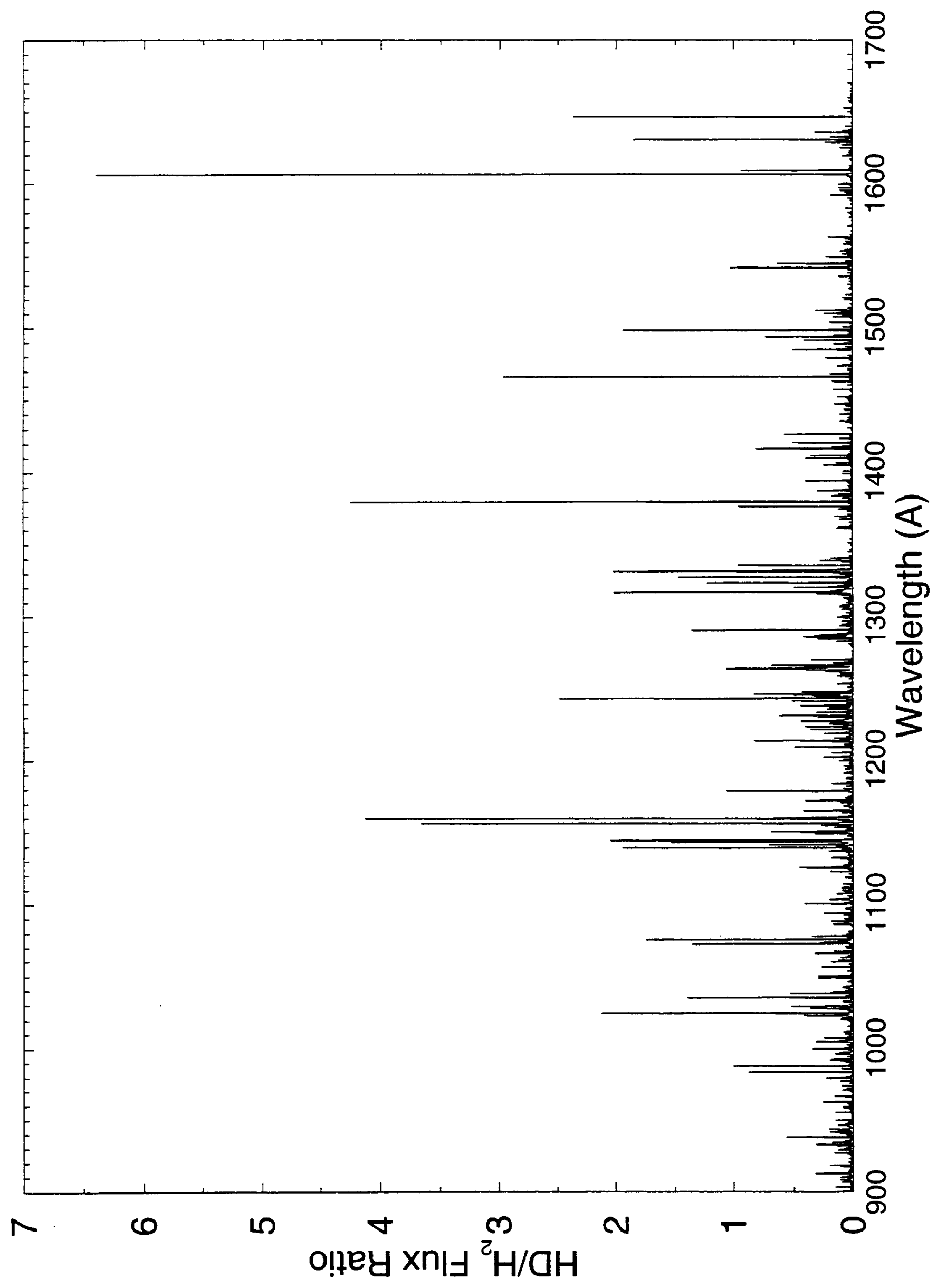




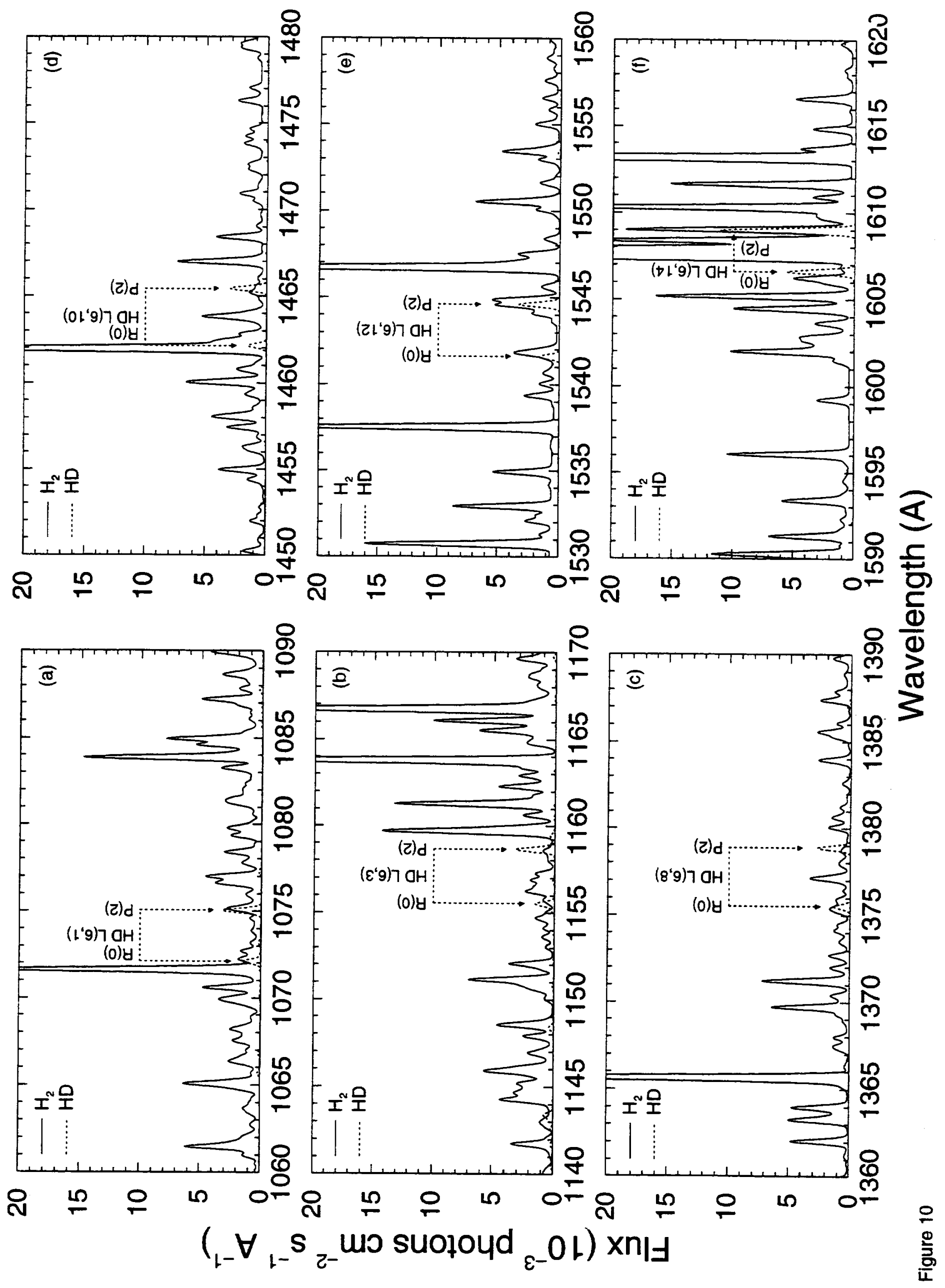

